\title{
QUASI-SEMISIMPLE ELEMENTS
}

\author{
F. DIGNE AND J. MICHEL
}

\begin{abstract}
We study quasi-semisimple elements of disconnected reductive algebraic groups over an algebraically closed field. We describe their centralizers, define isolated and quasi-isolated quasi-semisimple elements and classify their conjugacy classes.
\end{abstract}

\section{INTRODUCTION}

In this paper we study conjugacy classes and centralizers of quasi-semisimple elements in disconnected reductive groups over algebraically closed fields.

Let $\mathbf{G}$ be an algebraic group such that the connected component $\mathbf{G}^{0}$ is reductive. Let $\mathbf{G}^{1}$ be a connected component of $\mathbf{G}$ and $\mathbf{H}$ be the subgroup of $\mathbf{G}$ generated by $\mathbf{G}^{1}$. Then $\mathbf{G}^{0}$-conjugacy classes of $\mathbf{G}^{1}$ coincide with $\mathbf{H}$-conjugacy classes, and $\mathbf{G}$ conjugacy classes of $\mathbf{G}^{1}$ are unions of orbits of the finite group $\mathbf{G} / \mathbf{H}$ on $\mathbf{H}$-conjugacy classes. Thus it is reasonable to first consider the case where $\mathbf{G}$ is generated by $\mathbf{G}^{1}$.

This is closely related to the study of algebraic automorphisms of $\mathbf{G}^{0}$ : if $\mathbf{G}^{0}$ is adjoint, the elements of $\mathbf{G}^{1}$ can be interpreted as a class of automorphisms of $\mathbf{G}^{0}$ modulo inner automorphisms.

According to these considerations, in what follows we will use the following setting: we denote by $\sigma$ an element of a (disconnected) reductive group with identity component denoted by $\mathbf{G}$, and we study the conjugacy classes in the coset $\mathbf{G}^{1}:=$ $\mathbf{G} \cdot \sigma$.

We consider algebraic groups over some algebraically closed field $k$ of characteristic $p$, where $p$ is 0 or a prime number.

We denote by $n_{\sigma}$ the smallest power of $\sigma$ which acts by an inner automorphism on $\mathbf{G}$.

A quasi-semisimple element is an element of $\mathbf{G}^{1}$ which stabilizes a pair $\mathbf{T} \subset \mathbf{B}$ of a maximal torus of $\mathbf{G}^{0}$ and a Borel subgroup containing it; let $\mathbf{T}^{1}$ be the normalizer of this pair in $\mathbf{G}^{1}$. Since all such pairs are conjugate, and we are interested in quasisemisimple elements up to conjugacy, we study the elements of $\mathbf{T}^{1}$.

An element $\sigma \in \mathbf{T}^{1}$ acts on $W:=N_{\mathbf{G}}(\mathbf{T}) / \mathbf{T}$; its order as an automorphism of $\mathbf{T}$ is $n_{\sigma}$. If $\mathbf{G}$ is semisimple, $n_{\sigma}$ is also the order of the action of $\sigma$ on $W$ since an algebraic automorphism of $\mathbf{G}$ stabilizing $\mathbf{T}$ and trivial on $W$ is inner.

Our results are as follows.

In the first section, we study the centralizer $\mathbf{G}^{\sigma}$ of $\sigma$, generalizing the work of Steinberg [St68] when $\mathbf{G}$ is semisimple simply connected, and of DM94 which deals with the connected component $\left(\mathbf{G}^{\sigma}\right)^{0}$. Then we give a first approach to

2010 Mathematics Subject Classification. 20G15.

Key words and phrases. disconnected reductive groups, quasi-semisimple automorphisms, quasi-isolated classes. 
the classification of semisimple classes, reducing the problem to questions on the quotient torus $\mathbf{T} /[\mathbf{T}, \sigma]$.

In the second section, we fix a quasi-central element $\sigma$ (see Definition 1.19) to give more specific results: we need only to consider elements $t \sigma \in\left(\mathbf{T}^{\sigma}\right)^{0} \cdot \sigma$, and we introduce a root system $\Phi_{\sigma}$ such that quasi-semisimple classes are classified by elements of the fundamental alcove of the corresponding affine Weyl group.

In the third section we introduce isolated and quasi-isolated elements and indicate a method to classify them. This allows us in particular to state Theorem 3.11 which describes the exponent of the group $Z\left(\left(\mathbf{G}^{t \sigma}\right)^{0}\right) / Z^{0}\left(\left(\mathbf{G}^{t \sigma}\right)^{0}\right)$.

Finally in the last section we classify explicitly the quasi-isolated classes in quasisimple reductive groups, and describe their centralizers.

The assumption that $k$ is algebraically closed is unnecessary for quite a few results in this paper, but we adopted it for simplicity.

We thank Cedric Bonnafé twice. First, for having written [B05] which we generalize to our setting. Second, for giving us the impulse of writing this paper by asking us whether a result like Theorem 3.11 could be true. We thank Gunter Malle for his remarks on a first version of this paper.

\section{Quasi-SEMisimple Elements}

The tori $\left(\mathbf{T}^{\sigma}\right)^{0}$ and $\mathbf{T} /[\mathbf{T}, \sigma]$. In this subsection, we describe facts valid for any torus $\mathbf{T}$ with an algebraic automorphism $\sigma$ of finite order $n$.

Let $Y:=Y(\mathbf{T})=\operatorname{Hom}\left(k^{\times}, \mathbf{T}\right)$ and $X:=X(\mathbf{T})=\operatorname{Hom}\left(\mathbf{T}, k^{\times}\right)$, and let $($, denote the natural pairing between $Y$ and $X$.

We have $Y\left(\left(\mathbf{T}^{\sigma}\right)^{0}\right)=Y^{\sigma}$. To describe $X\left(\left(\mathbf{T}^{\sigma}\right)^{0}\right)$ we introduce the map $\pi$ : $X \otimes \mathbb{Q} \rightarrow X \otimes \mathbb{Q}: x \mapsto n^{-1} \sum_{i=1}^{n} \sigma^{i}(x)$. Then

Lemma 1.1. The restriction of characters from $\mathbf{T}$ to $\left(\mathbf{T}^{\sigma}\right)^{0}$ induces an isomorphism $\pi(X) \stackrel{\sim}{\rightarrow} X\left(\left(\mathbf{T}^{\sigma}\right)^{0}\right)$. The natural action of $W^{\sigma}$ on $X\left(\left(\mathbf{T}^{\sigma}\right)^{0}\right)$ corresponds via this isomorphism to the restriction of its action to $\pi(X) \subset X \otimes \mathbb{Q}$.

Proof. For $t \in\left(\mathbf{T}^{\sigma}\right)^{0}$ and $\chi \in X$ all the elements in the $\sigma$-orbit of $\chi$ have same value on $t$, hence the value $\chi(t)$ depends only on $\pi(\chi)$. Hence, if we show that ker $\pi$ coincides with the characters which have a trivial restriction to $\left(\mathbf{T}^{\sigma}\right)^{0}$, we can factorize $\operatorname{Res}_{\left(\mathbf{T}^{\sigma}\right)^{0}}^{\mathbf{T}}$ through an injective $W^{\sigma}$-equivariant map $\pi(X) \stackrel{\iota}{\rightarrow} X\left(\left(\mathbf{T}^{\sigma}\right)^{0}\right)$. The image of the map $N_{\sigma}: \mathbf{T} \rightarrow \mathbf{T}: t \mapsto t \cdot{ }^{\sigma} t \ldots{ }^{\sigma^{n-1}} t$ is $\left(\mathbf{T}^{\sigma}\right)^{0}$ since the image is a torus, consists of $\sigma$-stable elements, and $N_{\sigma}$ acts on $\left(\mathbf{T}^{\sigma}\right)^{0}$ as raising to the $n$-th power. A character $\chi \in X$ is in $\operatorname{ker} \pi$ if and only if $0=\left(1+\sigma+\ldots+\sigma^{n-1}\right) \chi=\chi \circ N_{\sigma}$ which is equivalent to $\operatorname{Res}_{\left(\mathbf{T}^{\sigma}\right)^{0}}^{\mathbf{T}} \chi=0$.

It remains to show that $\iota$ is surjective; this comes from the fact that any character of the subtorus $\left(\mathbf{T}^{\sigma}\right)^{0}$ can be lifted to a character of $\mathbf{T}$, since a subtorus is a direct factor.

Lemma 1.2. (1) $\mathbf{T}^{\sigma} /\left(\mathbf{T}^{\sigma}\right)^{0}$ is isomorphic to the $p^{\prime}$-part of the group

$$
H=\operatorname{Ker}\left(1+\sigma+\ldots+\sigma^{n-1} \mid X\right) /(\sigma-1) X,
$$

whose exponent divides $n$.

(2) If $\sigma$ permutes a basis of $X$, then $\operatorname{Ker}\left(1+\sigma+\ldots+\sigma^{n-1} \mid X\right)=(\sigma-1) X$. Thus if $\sigma$ permutes a basis of $X$ then $\mathbf{T}^{\sigma}=\left(\mathbf{T}^{\sigma}\right)^{0}$. 
(3) The intersection $[\mathbf{T}, \sigma] \cap \mathbf{T}^{\sigma}$ has exponent $n$, where $[\mathbf{T}, \sigma]$ is the torus $\left\{t \sigma\left(t^{-1}\right) \mid t \in \mathbf{T}\right\}$. We have $\mathbf{T}=[\mathbf{T}, \sigma] \cdot\left(\mathbf{T}^{\sigma}\right)^{0}$, and $Y([\mathbf{T}, \sigma])=\operatorname{Ker}(1+$ $\left.\sigma+\ldots+\sigma^{n-1} \mid Y\right)$.

Proof. For (1), $\mathbf{T}^{\sigma} /\left(\mathbf{T}^{\sigma}\right)^{0}$ is isomorphic to $X\left(\mathbf{T}^{\sigma}\right)_{p^{\prime} \text {-tors }}$, where $p^{\prime}$-tors denotes the $p^{\prime}$-part of the torsion. Considering the map $t \mapsto[t, \sigma]: \mathbf{T} \rightarrow[\mathbf{T}, \sigma]$ of kernel $\mathbf{T}^{\sigma}$ and using the exactness of the functor $X$, we get that $X\left(\mathbf{T}^{\sigma}\right)_{p^{\prime} \text {-tors }}$ is isomorphic to $(X /(\sigma-1) X)_{p^{\prime} \text {-tors. }}$ Since $X / \operatorname{Ker}\left(1+\sigma+\ldots+\sigma^{n-1}\right)$ has no torsion, we get that $X\left(\mathbf{T}^{\sigma}\right)_{p^{\prime} \text {-tors }}$ is isomorphic to $H_{p^{\prime} \text {-tors }}$. Now modulo $\sigma-1$ we have $(1+\sigma+\ldots+$ $\left.\sigma^{n-1}\right) x=n x$ whence the assertion that the exponent of $H$ divides $n$.

(2) results from an explicit computation: let $x_{1}, \ldots, x_{r}$ be a basis of $X$ such that $\sigma$ induces a permutation of $\{1, \ldots, r\}$. Then $\sum_{i} a_{i} x_{i} \in \operatorname{Ker}\left(1+\sigma+\ldots+\sigma^{n-1}\right)$ if and only if for each orbit $\mathcal{O}$ of $\sigma$ on $\{1, \ldots, r\}$ we have $\sum_{i \in \mathcal{O}} a_{i}=0$. On the other hand $\sum_{i} a_{i} x_{i}$ is in the image of $\sigma-1$ if and only if $a_{i}$ is of the form $b_{\sigma^{-1}(i)}-b_{i}$ for some sequence $b_{i}$. The two conditions are clearly equivalent.

The first item of (3) results from the remark that if $[t, \sigma] \in \mathbf{T}^{\sigma}$ then $[t, \sigma]^{n}=$ $\left(t \sigma\left(t^{-1}\right)\right) \sigma\left(t \sigma\left(t^{-1}\right)\right) \ldots \sigma^{n-1}\left(t \sigma\left(t^{-1}\right)\right)=t \sigma^{n}\left(t^{-1}\right)$. The second item follows: since the kernel of $t \mapsto[t, \sigma]: \mathbf{T} \rightarrow[\mathbf{T}, \sigma]$ is $\mathbf{T}^{\sigma}$, we have $\operatorname{dim} \mathbf{T}=\operatorname{dim}\left(\mathbf{T}^{\sigma}\right)^{0}+\operatorname{dim}[\mathbf{T}, \sigma]$ and the intersection is finite by the first part. Finally, for the third item, $Y([\mathbf{T}, \sigma])$ is a pure sublattice of $Y$ which spans the same $\mathbb{Q}$-subspace as the image of $\sigma-1$; this is the case of $\operatorname{Ker}\left(1+\sigma+\ldots+\sigma^{n-1} \mid Y\right)$, since a kernel is always a pure sublattice.

When $\sigma$ permutes a basis of $X$, by (2) of Lemma 1.2 $\pi(X)$ coincides with the $\sigma$-coinvariants of $X$. Though this assumption does not hold in general, the duality between $Y^{\sigma}$ and $\pi(X)$ plays a similar role to the duality between invariants and coinvariants. To remind of this analogy we will use the following notation:

Notation 1.3. We write $X_{\sigma}$ for $\pi(X)$ and $Y_{\sigma}$ for $\pi(Y)$ where $\pi: Y \rightarrow Y \otimes \mathbb{Q}$ : $y \mapsto n^{-1} \sum_{i=1}^{n} \sigma^{i}(y)$.

The following is true in general for lattices with a $\sigma$-stable pairing

Lemma 1.4. The restriction of the duality between $X \otimes \mathbb{Q}$ and $Y \otimes \mathbb{Q}$ to $(X \otimes \mathbb{Q})^{\sigma}$ and $(Y \otimes \mathbb{Q})^{\sigma}$ is a duality which makes the lattices $\left(X_{\sigma}, Y^{\sigma}\right)$, as well as $\left(X^{\sigma}, Y_{\sigma}\right)$, dual to each other.

Proof. That the restriction of the duality is a duality is clear.

Let us compute the dual of $X_{\sigma}$. If $y \in(Y \otimes \mathbb{Q})^{\sigma}$ and $(y, \pi(x)) \in \mathbb{Z}$ for all $x \in X$, then, since $($,$) is \sigma$-invariant we have $(y, \pi(x))=(y, x) \in \mathbb{Z}$ for all $x \in X$, so that $y \in Y$, hence $y \in Y^{\sigma}$. Conversely, if $y \in Y^{\sigma}$, then $(y, \pi(x)) \in \mathbb{Z}$ for all $x \in X$.

The proof for $\left(X^{\sigma}, Y_{\sigma}\right)$ is symmetric to the above proof.

Lemma 1.5. Through the isomorphism $\left(X\left(\left(\mathbf{T}^{\sigma}\right)^{0}\right), Y\left(\left(\mathbf{T}^{\sigma}\right)^{0}\right)\right) \simeq\left(X_{\sigma}, Y^{\sigma}\right)$ (see Lemma 1.1) the duality of Lemma 1.4 between $X_{\sigma}$ and $Y^{\sigma}$ identifies with the natural duality between $X\left(\left(\mathbf{T}^{\sigma}\right)^{0}\right)$ and $Y\left(\left(\mathbf{T}^{\sigma}\right)^{0}\right)$.

Proof. By definition, for $\lambda \in k, x \in X$ and $y \in Y$ we have $x(y(\lambda))=\lambda^{(y, x)}$. If $y \in$ $Y\left(\left(\mathbf{T}^{\sigma}\right)^{0}\right)=Y^{\sigma}$ then $x(y(\lambda))=\left(\operatorname{Res}_{\left(\mathbf{T}^{\sigma}\right)^{0}}^{\mathbf{T}}(x)\right)(y(\lambda))$, which proves the lemma.

Just as we identified $\left(X_{\sigma}, Y^{\sigma}\right)$ with $\left(X\left(\left(\mathbf{T}^{\sigma}\right)^{0}\right), Y\left(\left(\mathbf{T}^{\sigma}\right)^{0}\right)\right)$, the following lemma allows to identify $\left(X^{\sigma}, Y_{\sigma}\right)$ with $(X(\mathbf{T} /[\mathbf{T}, \sigma]), Y(\mathbf{T} /[\mathbf{T}, \sigma]))$.

Lemma 1.6. 
(1) The morphism $\mathbf{T} \rightarrow \mathbf{T} /[\mathbf{T}, \sigma]$ induces an isomorphism $X(\mathbf{T} /[\mathbf{T}, \sigma]) \simeq X^{\sigma}$.

(2) The natural map $f: Y \rightarrow Y(\mathbf{T} /[\mathbf{T}, \sigma])$ induces an isomorphism $Y_{\sigma} \simeq$ $Y(\mathbf{T} /[\mathbf{T}, \sigma])$.

(3) Through the above isomorphisms the natural duality between $X(\mathbf{T} /[\mathbf{T}, \sigma])$ and $Y(\mathbf{T} /[\mathbf{T}, \sigma])$ identifies with the duality of Lemma 1.4 between $X^{\sigma}$ and $Y_{\sigma}$.

Proof. A character $x \in X$ is trivial on $[\mathbf{T}, \sigma]$ if and only if $x$ is $\sigma$-stable. Since $X$ is exact this gives the isomorphism $X^{\sigma} \simeq X(\mathbf{T} /[\mathbf{T}, \sigma])$ of (1).

We now prove (2). The image $f(y) \in Y(\mathbf{T} /[\mathbf{T}, \sigma])$ depends only on $\pi(y)$ : indeed let $y \in \operatorname{ker} \pi$, then $n_{\sigma} y$ is in $\operatorname{ker} \pi$ so for $\lambda \in k^{\times}$we have $n_{\sigma} \pi(y)(\lambda)=1$, that is $N_{\sigma}(y(\lambda))=1$, where $N_{\sigma}$ is as in the proof of Lemma 1.1. Thus $y\left(k^{\times}\right)$is a subtorus of $\operatorname{ker} N_{\sigma}$ and the latter contains $[\mathbf{T}, \sigma]$ as a finite index subgroup, thus $y\left(k^{\times}\right) \subset[\mathbf{T}, \sigma]$. Conversely, if $y\left(k^{\times}\right) \subset[\mathbf{T}, \sigma]$ then $N_{\sigma} \circ y$ is trivial, so $y \in \operatorname{ker} \pi$. Hence we get a well defined injective map $Y_{\sigma} \rightarrow Y(\mathbf{T} /[\mathbf{T}, \sigma])$. The surjectivity comes from the fact that, being a subtorus $[\mathbf{T}, \sigma]$ is a direct factor of $\mathbf{T}$, so that a cocharacter of $\mathbf{T} /[\mathbf{T}, \sigma]$ can be lifted to $\mathbf{T}$.

We now prove (3). The pairing between elements of $X(\mathbf{T} /[\mathbf{T}, \sigma])$ and $Y(\mathbf{T} /[\mathbf{T}, \sigma])$ identified via (1) and (2) respectively with $x \in X^{\sigma}$ and $y \in Y_{\sigma}$ is given by the computation of $\lambda \mapsto x(y(\lambda) \bmod [T, \sigma])=x(y(\lambda))=\lambda^{(y, x)}$. Since $(y, x)=(\pi(y), x)$ by $\sigma$-invariance of the pairing and of $x$, we get (3).

We note for future reference the following:

Lemma 1.7. $\left(\left(\mathbf{T} /\left(\mathbf{T}^{\sigma}\right)^{0}\right)^{\sigma}\right)^{0}=\{1\}$.

Proof. By Lemma 1.1 the restriction map from $X(\mathbf{T})$ to $X\left(\left(\mathbf{T}^{\sigma}\right)^{0}\right)$ is $\pi$. From the exactness of the functor $X$ we deduce $X\left(\mathbf{T} /\left(\mathbf{T}^{\sigma}\right)^{0}\right)=\operatorname{ker} \pi$. Applying Lemma 1.1 again we get $X\left(\left(\left(\mathbf{T} /\left(\mathbf{T}^{\sigma}\right)^{0}\right)^{\sigma}\right)^{0}\right)=\pi(\operatorname{ker}(\pi))=\{0\}$, whence the result.

Centralizers of quasi-semisimple elements. Let as in the introduction $\sigma \in \mathbf{G}^{1}$ be a quasi-semisimple element stabilizing the pair $\mathbf{T} \subset \mathbf{B}$ (thus $\sigma \in \mathbf{T}^{1}$ ).

Let $\Sigma$ be the root system of $\mathbf{G}$ with respect to $\mathbf{T}$ and $\Sigma^{\vee}$ be the corresponding set of coroots. The coroot corresponding to $\alpha \in \Sigma$ will be denoted by $\alpha^{\vee}$. The $\sigma$-stable Borel subgroup B determines a $\sigma$-stable order on $\Sigma$ and we denote by $\Sigma^{+}$ and $\Pi$ respectively the positive and the simple roots for this order. The element $\sigma$ induces a permutation of $\Pi$ and $\Sigma$.

We recall (see for instance DM94, 1.8(v)]) that, if $x_{\alpha}: \mathbb{G}_{a} \stackrel{\sim}{\rightarrow} \mathbf{U}_{\alpha}$ is an isomorphism to the root subgroup of $\mathbf{G}$ attached to $\alpha$, and $\mathcal{O}$ is the orbit under $\sigma$ of $\alpha$, there is a unique $C_{\sigma, \mathcal{O}} \in k^{\times}$(depending only on $\mathcal{O}$ and not on $\alpha$ ) such that ${ }^{\sigma^{|\mathcal{O}|}} x_{\alpha}(\lambda)=x_{\alpha}\left(C_{\sigma, \mathcal{O}} \lambda\right)$ for any $\lambda \in k$.

We introduce the following terminology:

Definition 1.8. We say that the $\sigma$-orbit $\mathcal{O} \subset \Sigma$ is special if there exist two roots $\alpha, \beta \in \mathcal{O}$ such that $\alpha+\beta \in \Sigma$. In this case, we say that the orbit of $\alpha+\beta$ is cospecial.

Remark 1.9. Special orbits only exist if $\mathbf{G}$ has a component of type $A_{2 r}$. If $\alpha$ and $\beta$ are distinct roots in the same $\sigma$-orbit then $\left(\alpha^{\vee}, \beta\right)=0$ except if the orbit is special and $\alpha+\beta$ is a root, in which case $\left(\alpha^{\vee}, \beta\right)=-1$.

Definition 1.10. Let $\mathcal{O}(\alpha)$ denote the orbit of $\alpha$ under $\sigma$. 
- If $p \neq 2$ we define $\Sigma_{\sigma}:=\left\{\pi(\alpha) \mid \alpha \in \Sigma, C_{\sigma, \mathcal{O}(\alpha)}=1\right\} \subset X_{\sigma}$. If $p=2$ we define $\Sigma_{\sigma}:=\left\{\pi(\alpha) \mid \alpha \in \Sigma, C_{\sigma, \mathcal{O}(\alpha)}=1, \mathcal{O}(\alpha)\right.$ not special. $\}$.

- We define $\Sigma_{\sigma}^{\vee}:=\left\{\bar{\alpha}^{\vee} \mid \pi(\alpha) \in \Sigma_{\sigma}, \mathcal{O}(\alpha)\right.$ not special $\} \cup\left\{2 \bar{\alpha}^{\vee} \mid \pi(\alpha) \in\right.$ $\Sigma_{\sigma}, \mathcal{O}(\alpha)$ special $\} \subset Y^{\sigma}$ where $\bar{\alpha}^{\vee}:=\sum_{\beta \in \mathcal{O}(\alpha)} \beta^{\vee}$.

\section{Proposition 1.11.}

(1) The group $\left(\mathbf{G}^{\sigma}\right)^{0}$ is reductive; its root system with respect to $\left(\mathbf{T}^{\sigma}\right)^{0}$ is $\Sigma_{\sigma}$, and the corresponding set of coroots is $\Sigma_{\sigma}^{\vee}$; we denote by $W^{0}(\sigma)$ the corresponding Weyl group.

(2) $W^{\sigma}$ acts on $X_{\sigma} \subset X \otimes \mathbb{Q}$ and on $Y^{\sigma}$ by restriction. $\left(W^{\sigma}, S_{\sigma}\right)$ is a Coxeter system where $S_{\sigma}$ is the set of $w_{\mathcal{O}}$ where $w_{\mathcal{O}}$ denotes the longest element of the parabolic subgroup $W_{\mathcal{O}}$, and $\mathcal{O}$ runs over the orbits of $\sigma$ in $S$.

(3) We have $N_{\mathbf{G}}(\mathbf{T})^{\sigma}=N_{\mathbf{G}^{\sigma}}\left(\left(\mathbf{T}^{\sigma}\right)^{0}\right)$ and $W^{0}(\sigma) \subset W^{\sigma}$ as subgroups of $\mathrm{GL}\left(X_{\sigma}\right)$.

(4) The set $\Sigma_{\sigma}^{+}:=\left\{\pi(\alpha) \in \Sigma_{\sigma} \mid \alpha \in \Sigma^{+}\right\}$is a set of positive roots in $\Sigma_{\sigma}$ corresponding to the Borel subgroup $\left(\mathbf{B}^{\sigma}\right)^{0}$ of $\left(\mathbf{G}^{\sigma}\right)^{0}$.

Proof. We prove (1). The fact that $\left(\mathbf{G}^{\sigma}\right)^{0}$ is reductive is in [DM94, Proposition 1.8]. The description of its root system is in [DM02, Proposition 2.1]. Let us describe the coroots. Let $\mathbf{U}_{\alpha}$ be the root subgroup of $\mathbf{G}$ normalized by $\mathbf{T}$ on which $\mathbf{T}$ acts by $\alpha \in \Sigma$. For $\left\{\alpha \in \Sigma \mid \pi(\alpha) \in \Sigma_{\sigma}\right\}$ we may choose a set of isomorphisms $u_{\alpha}: \mathbb{G}_{a} \stackrel{\sim}{\rightarrow}$ $\mathbf{U}_{\alpha}$ such that $u_{\sigma(\alpha)}={ }^{\sigma}\left(u_{\alpha}\right)$. Then if $\mathcal{O}(\alpha)$ is not special, the root subgroup of $\left(\mathbf{G}^{\sigma}\right)^{0}$ on which $\mathbf{T}^{\sigma}$ acts by the restriction of $\alpha$ is the image of $u_{\pi(\alpha)}:=\prod_{i} u_{\sigma^{i}(\alpha)}$. Then the general formula $\beta^{\vee}(\lambda)=u_{\beta}(\lambda-1) u_{-\beta}(1) u_{\beta}\left(\lambda^{-1}-1\right) u_{-\beta}(-\lambda)$ applied with $\beta=\pi(\alpha)$ gives $\pi(\alpha)^{\vee}=\bar{\alpha}^{\vee}$ using that $\mathbf{U}_{\alpha}$ and $\mathbf{U}_{ \pm \alpha^{\prime}}$ commute for all $\alpha \neq \alpha^{\prime}$ in the same $\sigma$-orbit, by Remark 1.9 .

If $\mathcal{O}(\alpha)$ is special (which implies $p \neq 2$ ), then $\alpha+\sigma^{j}(\alpha)$ is a root for some $j$. As the following computation shows, the root subgroup of $\left(\mathbf{G}^{\sigma}\right)^{0}$ on which $\mathbf{T}^{\sigma}$ acts by $\pi(\alpha)$ is isomorphic to the fixed points of $\sigma^{j}$ on the subgroup $\left\langle\mathbf{U}_{\alpha}, \mathbf{U}_{\sigma^{j}(\alpha)}\right\rangle$. The subgroup $\mathbf{G}_{\alpha}=\left\langle\mathbf{U}_{ \pm \alpha}, \mathbf{U}_{ \pm \sigma^{j}(\alpha)}\right\rangle$ is isomorphic to $\mathrm{SL}_{3}$ or $\mathrm{PGL}_{3}$. For a certain choice of the morphisms $u_{ \pm\left(\alpha+\sigma^{j}(\alpha)\right)}$ the restriction of $\sigma^{j}$ to $\mathbf{G}_{\alpha}$ is the action of transpose $\circ$ inverse $\circ$ ad $\left(\begin{array}{ccc}0 & 0 & -2 \\ 0 & 1 & 0 \\ -2 & 0 & 0\end{array}\right)$ on $3 \times 3$ matrices. With this model, one checks that $\left(\mathbf{G}_{\alpha}^{\sigma}\right)^{0}$ is isomorphic to the image of $\mathrm{SL}_{2}$ by the rational representation expressing the action of $\mathrm{SL}_{2}$ on homogeneous polynomials of degree two. If the matrix $\left(\begin{array}{ll}a & b \\ c & d\end{array}\right)$ acts by $X \mapsto a X+c Y, Y \mapsto b X+d Y$, on the basis $\left\{X^{2}, X Y, Y^{2}\right\}$ it acts by $\left(\begin{array}{ccc}a^{2} & a b & b^{2} \\ 2 a c & a d+b c & 2 b d \\ c^{2} & c d & d^{2}\end{array}\right)$. It is readily seen on this model that the coroot of $\left(\mathbf{G}^{\sigma}\right)^{0}$, the image of the coroot of $\mathrm{SL}_{2}$, is equal to $2 \bar{\alpha}^{\vee}$.

Let us prove (2). The action of $W^{\sigma}$ on $X_{\sigma}$ has been explained in Lemma 1.1 The other facts about $\left(W^{\sigma}, S_{\sigma}\right)$ are proved in [St68, 1.32].

We prove (3). By [DM94, 1.8(iv)] $N_{\mathbf{G}}\left(\left(\mathbf{T}^{\sigma}\right)^{0}\right) \subset N_{\mathbf{G}}(\mathbf{T})$, thus $N_{\left(\mathbf{G}^{\sigma}\right)^{0}}\left(\left(\mathbf{T}^{\sigma}\right)^{0}\right)$ embeds into $N_{\mathbf{G}}(\mathbf{T})^{\sigma}$. Conversely a $\sigma$-fixed element which normalizes $\mathbf{T}$ normalizes $\mathbf{T}^{\sigma}$ hence normalizes its connected component $\left(\mathbf{T}^{\sigma}\right)^{0}$. Whence the first assertion of (3), and the fact that $W^{0}(\sigma)$ embeds into $W^{\sigma}$. 
That $\left(\mathbf{B}^{\sigma}\right)^{0}$ is a Borel subgroup of $\left(\mathbf{G}^{\sigma}\right)^{0}$ is DM94, 1.8 (iii)]. The set $\Sigma_{\sigma}^{+}$is a set of positive roots for the order induced by $\Sigma^{+}$. The corresponding root subgroups are subgroups of $\mathbf{B}$ as seen in the proof of (1), whence (4).

\section{Connected components of centralizers.}

Proposition 1.12. We have $\mathbf{G}^{\sigma} /\left(\mathbf{G}^{\sigma}\right)^{0} \simeq N_{\mathbf{G}}(\mathbf{T})^{\sigma} / N_{\left(\mathbf{G}^{\sigma}\right)^{0}}\left(\left(\mathbf{T}^{\sigma}\right)^{0}\right)$

Proof. We first show that $\mathbf{G}^{\sigma}$ is generated by $\left(\mathbf{G}^{\sigma}\right)^{0}$ and $\sigma$-stable representatives in $N_{\mathbf{G}}(\mathbf{T})$ of $W^{\sigma}$. A double coset $\mathbf{B} w \mathbf{B}$ containing an $x \in \mathbf{G}^{\sigma}$ is $\sigma$-stable, hence $w$ is $\sigma$-stable. Choose a representative $\dot{w}$ of $w$ and write $x$ uniquely as $x=u t_{1} \dot{w} u_{1}$ with $u \in \mathbf{U} \cap{ }^{w} \mathbf{U}^{-}, t_{1} \in \mathbf{T}$ and $u_{1} \in \mathbf{U}$, where $\mathbf{U}$ is the unipotent radical of $\mathbf{B}$. By uniqueness, $u$ and $u_{1}$ are in $\mathbf{U}^{\sigma} \subset\left(\mathbf{G}^{\sigma}\right)^{0}$ (the inclusion by [DM94, 1.8(i)]) and $t_{1} \dot{w}$ is $\sigma$-stable, so we get that $\mathbf{G}^{\sigma} /\left(\mathbf{G}^{\sigma}\right)^{0}$ has representatives in $N_{\mathbf{G}}(\mathbf{T})^{\sigma}$.

Since by Proposition $1.11(3)$, we have $N_{\mathbf{G}}(\mathbf{T})^{\sigma} \cap\left(\mathbf{G}^{\sigma}\right)^{0}=N_{\left(\mathbf{G}^{\sigma}\right)^{0}}\left(\left(\mathbf{T}^{\sigma}\right)^{0}\right)$, we get the proposition.

Notation 1.13. Let $W(\sigma):=\left\{w \in W^{\sigma} \mid[\sigma, \dot{w}] \in[\mathbf{T}, \sigma]\right\}$ for some representative $\dot{w}$ of $w$.

Note that the condition does not depend on the representative $\dot{w}$ of $w$ chosen.

Proposition 1.14. The natural morphisms give an exact sequence

$$
1 \rightarrow \mathbf{T}^{\sigma} /\left(\mathbf{T}^{\sigma}\right)^{0} \rightarrow N_{\mathbf{G}}(\mathbf{T})^{\sigma} / N_{\left(\mathbf{G}^{\sigma}\right)^{0}}\left(\left(\mathbf{T}^{\sigma}\right)^{0}\right) \rightarrow W(\sigma) / W^{0}(\sigma) \rightarrow 1
$$

Proof. Observe first that $W(\sigma)$ is the set of elements of $W^{\sigma}$ which have representatives in $N_{\mathbf{G}}(\mathbf{T})^{\sigma}$ (thus in $N_{\mathbf{G}^{\sigma}}\left(\left(\mathbf{T}^{\sigma}\right)^{0}\right)$ ); indeed $[\sigma, \dot{w}] \in[\mathbf{T}, \sigma]$ can be written ${ }^{\sigma} \dot{w} \dot{w}^{-1}={ }^{\sigma} t_{1}^{-1} t_{1}$ for some $t_{1} \in \mathbf{T}$ which is equivalent to ${ }^{\sigma}\left(t_{1} \dot{w}\right)=t_{1} \dot{w}$.

This observation can be rephrased as the exact sequence

$$
1 \rightarrow \mathbf{T}^{\sigma} \rightarrow N_{\mathbf{G}}(\mathbf{T})^{\sigma} \rightarrow W(\sigma) \rightarrow 1
$$

By Proposition 1.12 we have $N_{\mathbf{G}}(\mathbf{T})^{\sigma} \cap\left(\mathbf{G}^{\sigma}\right)^{0}=N_{\left(\mathbf{G}^{\sigma}\right)^{0}}\left(\left(\mathbf{T}^{\sigma}\right)^{0}\right)$ which is a normal subgroup of $N_{\mathbf{G}}(\mathbf{T})^{\sigma}$. Taking the intersections with $\left(\mathbf{G}^{\sigma}\right)^{0}$ and using that the image in $W(\sigma)$ of $N_{\left(\mathbf{G}^{\sigma}\right)^{0}}\left(\left(\mathbf{T}^{\sigma}\right)^{0}\right)$ is $W^{0}(\sigma)$, we get the exact sequence of the proposition.

Classification of quasi-semisimple elements. We want to study G-orbits (conjugacy classes) of quasi-semisimple elements of $\mathbf{G}^{1}$, and their centralizers in $\mathbf{G}$. We say that two quasi-semisimple elements have same type if they have conjugate centralizers, and we want to classify the possible types of quasi-semisimple elements. We can reduce this study to elements of finite order, thanks to the:

Lemma 1.15. For any $\sigma \in \mathbf{T}^{1}$ there exists an element $\sigma^{\prime} \in \sigma \cdot\left(\mathbf{T}^{\sigma}\right)^{0}$ of finite order such that $\mathbf{G}^{\sigma}=\mathbf{G}^{\sigma^{\prime}}$.

Proof. Let $\mathbf{S}$ be the Zariski closure of $\langle\sigma\rangle$ in $\langle\sigma\rangle \cdot\left(\mathbf{T}^{\sigma}\right)^{0}$. We have $\mathbf{S}^{0} \subset\left(\mathbf{T}^{\sigma}\right)^{0}$, so $\mathbf{S}^{0}$ is diagonalizable, and $\mathbf{S} / \mathbf{S}^{0}$ is generated by the image of $\sigma$. By the proof of [B05, Lemma 1.6], which uses only that $\mathbf{S}^{0}$ is diagonalizable, the generators of $\mathbf{S} / \mathbf{S}^{0}$ can be lifted to finite order elements of $\mathbf{S}$ and for any such $d \in \mathbf{S}$, there exists $t \in \mathbf{S}^{0}$ such that $t d$ has finite order and satisfies $C_{\mathbf{G}}(t d)=C_{\mathbf{G}}(\mathbf{S})=\mathbf{G}^{\sigma}$. If we take $d$ lifting $\sigma$ we get a finite order element $\sigma^{\prime}=t d \in \sigma \cdot\left(\mathbf{T}^{\sigma}\right)^{0}$, which has same centralizer as $\sigma$. 
To classify quasi-semisimple classes, we can fix an element $\sigma \in \mathbf{T}^{1}$ of finite order and write the others as $t \sigma$ with $t \in \mathbf{T}$. Note that two elements $t \sigma, t \sigma^{\prime}$ of $\mathbf{T}^{1}$ are $\mathbf{G}$-conjugate if and only if $t$ and $t^{\prime}$ are $\sigma$-conjugate under $\mathbf{G}$, where $\sigma$-conjugacy is the "twisted conjugacy" $t \mapsto g^{-1} t \sigma(g)$.

\section{Proposition 1.16.}

(1) Quasi-semisimple classes of $\mathbf{G}^{1}$ have representatives in $\left(\mathbf{T}^{\sigma}\right)^{0} \cdot \sigma$.

(2) The $\mathbf{G}$-conjugacy class of $t \sigma \in\left(\mathbf{T}^{\sigma}\right)^{0} \cdot \sigma$ (or equivalently the $\sigma$-conjugacy class of $t$ ) is parameterized by the $W^{\sigma}$-orbit of the image of $t$ in $\mathbf{T} /[\mathbf{T}, \sigma]=$ $\left(\mathbf{T}^{\sigma}\right)^{0} /\left([\mathbf{T}, \sigma] \cap\left(\mathbf{T}^{\sigma}\right)^{0}\right)$; the group $W^{\sigma}$ acts on $\mathbf{T} /[\mathbf{T}, \sigma]$ since it stabilizes $[\mathbf{T}, \sigma]$.

(3) Given $t \in\left(\mathbf{T}^{\sigma}\right)^{0}$, there exists $t^{\prime} \in\left(\mathbf{T}^{\sigma}\right)^{0}$, of finite order, such that $C_{\mathbf{G}}\left(t^{\prime} \sigma\right)=$ $C_{\mathbf{G}}(t \sigma)$.

Proof. (1) and (2) are explained in [DM15, 7.1, 7.2]. They use that $\mathbf{T}=[\mathbf{T}, \sigma]$. $\left(\mathbf{T}^{\sigma}\right)^{0}$.

For (3) we use Lemma 1.15 which constructs a $t^{\prime} \sigma \in \sigma\left(\mathbf{T}^{\sigma}\right)^{0}$ of finite order such that $C_{\mathbf{G}}\left(t^{\prime} \sigma\right)=C_{\mathbf{G}}(t \sigma)$. Since $\sigma$ commutes to $t^{\prime}$ and is of finite order, it is equivalent that $t^{\prime} \sigma$ be of finite order or $t^{\prime}$ be of finite order.

By Proposition1.16(1), quasi-semisimple classes have representatives $t \sigma \in\left(\mathbf{T}^{\sigma}\right)^{0}$. $\sigma$ where $\left(\mathbf{T}^{\sigma}\right)^{0}$ is a maximal torus of $\left(\mathbf{G}^{t \sigma}\right)^{0}$ as well as of $\left(\mathbf{G}^{\sigma}\right)^{0}$.

In the following, we will describe representatives of the conjugacy classes of quasi-semisimple elements of finite order and describe their centralizer, which by Proposition 1.16(3) suffices to classify all types of quasi-semisimple elements.

Theorem 1.17. The G-conjugacy classes of quasi-semisimple elements of finite order in $\mathbf{G}^{1}$ are parameterized by the orbits in $Y_{\sigma} \otimes \mathbb{Q}_{p^{\prime}}$ of the extended affine Weyl group $\widetilde{W}:=Y_{\sigma} \rtimes W^{\sigma}$, where the action of $W^{\sigma}$ is as in Proposition 1.16(2).

To describe this parameterization, we fix an isomorphism $(\mathbb{Q} / \mathbb{Z})_{p^{\prime}} \stackrel{\sim}{\rightarrow} \mu(k)$, which lifts to a map $\iota: \mathbb{Q}_{p^{\prime}} \rightarrow \mu(k)$, where $\mu(k)$ is the group of roots of unity in $k$. Then the $\widetilde{W}$-orbit of $\lambda \otimes x$, where $\lambda \in Y_{\sigma}$ and $x \in \mathbb{Q}_{p^{\prime}}$, defines $\lambda(\iota(x)) \in \mathbf{T} /[\mathbf{T}, \sigma]$ whose preimages in $\left(\mathbf{T}^{\sigma}\right)^{0}$ are $\sigma$-conjugate.

Proof. By Proposition 1.16(2), the quasi-semisimple classes of $\mathbf{G}^{1}$ are parameterized by the $W^{\sigma}$-orbits in $\mathbf{T} /[\mathbf{T}, \sigma]$. The map $\lambda \otimes x \mapsto \lambda(\iota(x))$ defines an isomorphism from $Y(\mathbf{T} /[\mathbf{T}, \sigma]) \otimes(\mathbb{Q} / \mathbb{Z})_{p^{\prime}}$ to the group of points of finite order of $\mathbf{T} /[\mathbf{T}, \sigma]$ (see for instance DM91, 0.20]). Since $Y(\mathbf{T} /[\mathbf{T}, \sigma])$ is equal to $Y_{\sigma}$ by Lemma 1.6 we get that the quasi-semisimple conjugacy classes of finite order are parameterized by elements of $Y_{\sigma} \otimes \mathbb{Q}_{p^{\prime}}$ up to action of $W^{\sigma}$ and translation by $Y_{\sigma}$. Whence the result.

Note that since the exponent of $[\mathbf{T}, \sigma] \cap\left(\mathbf{T}^{\sigma}\right)^{0}$ divides that of $\sigma$ (see Lemma $1.2(3))$, this group is formed of elements of finite order, thus identifies with a subset of $Y^{\sigma} \otimes \mathbb{Q} / \mathbb{Z}$.

\section{Proposition 1.18.}

(1) $Z(\mathbf{G})^{0} \subset[\mathbf{T}, \sigma]$ if and only if $\left(Z(\mathbf{G})^{\sigma}\right)^{0}=\{1\}$

(2) If the conditions of (1) hold the quasi-semisimple $\mathbf{G}$-orbits of $\mathbf{G}^{1}$ and the quasi-semisimple $\mathbf{G} / Z^{0}(\mathbf{G})$-orbits of $\mathbf{G}^{1} / Z^{0}(\mathbf{G})$ are in bijection by the quotient map. This bijection preserves $W(\sigma)$ and $W^{0}(\sigma)$. 
Proof. If $\left(Z(\mathbf{G})^{\sigma}\right)^{0}$ is trivial, since $Z(\mathbf{G})^{0}=\left(Z(\mathbf{G})^{\sigma}\right)^{0} \cdot\left[Z(\mathbf{G})^{0}, \sigma\right]$ by Lemma $1.2(3)$ applied to the torus $Z(\mathbf{G})^{0}$, we have $Z(\mathbf{G})^{0} \subset[\mathbf{T}, \sigma]$. Conversely if $\left.Z(\mathbf{G})^{0} \subset \mid \mathbf{T}, \sigma\right]$ then $\left(Z(\mathbf{G})^{\sigma}\right)^{0}$ is a connected subgroup of the finite group $\left.\left(\mathbf{T}^{\sigma}\right)^{0} \cap \mid \mathbf{T}, \sigma\right]$, hence is trivial. This proves (1).

Any quasi-semisimple class of $\mathbf{G}^{1}$ has a representative in $\mathbf{T}^{1}$. Two elements $\sigma, t \sigma \in \mathbf{T}^{1}$ have conjugate images $\bar{\sigma}, \overline{t \sigma}$ in $\mathbf{G}^{1} / Z^{0}(\mathbf{G})$ if and only if there exists $g \in \mathbf{G}$ such that $g \sigma g^{-1}=z t \sigma$ with $z \in Z^{0}(\mathbf{G})$. If the conditions of (1) are satisfied we can write $z=t_{1}^{-1 \sigma} t_{1}$ for some $t \in \mathbf{T}$, so that $t_{1} g \sigma g^{-1} t_{1}^{-1}=t \sigma$. Hence the quotient map induces a bijection on the classes.

Now for a representative $\dot{w}$ of $w \in W^{\sigma}$, the condition $[\bar{\sigma}, \bar{w}] \in[\overline{\mathbf{T}}, \bar{\sigma}]$ is equivalent to the condition $[\sigma, \dot{w}] \in[\mathbf{T}, \sigma]$, whence the preservation of $W(\sigma)$ by the quotient map. The preservation of $W^{0}(\sigma)$ comes from the fact that $\mathbf{G}$ and $\mathbf{G} / Z(\mathbf{G})^{0}$ have the same root systems and isomorphic root subgroups, thus same $C_{\sigma, \mathcal{O}(\alpha)}$ and $\Sigma_{\sigma}$.

Quasi-central elements. To classify quasi-semisimple elements it will be useful to relate them to quasi-central elements, whose definition we recall from DM94, 1.15]:

Definition 1.19. An element $\sigma$ is quasi-central if $\mathbf{G}^{\sigma}$ has maximal dimension among centralizers of elements of $\mathbf{G} \cdot \sigma$.

By [DM94, 1.15(iii)] we have:

Proposition 1.20. The following are equivalent:

(1) $\sigma$ is quasi-central.

(2) $\sigma$ is quasi-semisimple and $W^{\sigma}=W(\sigma)=W^{0}(\sigma)$.

By (2) above and Proposition 1.12, Proposition 1.14 reduces when $\sigma$ is quasicentral to $\mathbf{G}^{\sigma} /\left(\mathbf{G}^{\sigma}\right)^{0} \simeq \mathbf{T}^{\sigma} /\left(\mathbf{T}^{\sigma}\right)^{0}$.

Remark 1.21. When $\sigma$ is quasi-central, $\mathbf{T}^{\sigma}=\left(\mathbf{T}^{\sigma}\right)^{0} \cdot Z\left(\mathbf{G}^{\sigma}\right)$ and $\mathbf{T}^{\sigma} /\left(\mathbf{T}^{\sigma}\right)^{0}=$ $Z\left(\mathbf{G}^{\sigma}\right) / Z\left(\left(\mathbf{G}^{\sigma}\right)^{0}\right)$.

Proof. The second paragraph of the proof of [DM94, 1.29] gives the first assertion. It remains to show that $\left(\mathbf{T}^{\sigma}\right)^{0} \cap Z\left(\mathbf{G}^{\sigma}\right)=Z\left(\left(\mathbf{G}^{\sigma}\right)^{0}\right)$. We have $\left(\mathbf{T}^{\sigma}\right)^{0} \cap Z\left(\mathbf{G}^{\sigma}\right) \subset$ $Z\left(\left(\mathbf{G}^{\sigma}\right)^{0}\right)$. We get the reverse inclusion using $Z\left(\left(\mathbf{G}^{\sigma}\right)^{0}\right) \subset\left(\mathbf{T}^{\sigma}\right)^{0}$ so that $Z\left(\left(\mathbf{G}^{\sigma}\right)^{0}\right)$ commutes with $\left(\mathbf{G}^{\sigma}\right)^{0} . \mathbf{T}^{\sigma}$ which is equal to $\mathbf{G}^{\sigma}$ by the first paragraph of the proof of [DM94, 1.29].

Lemma 1.22. There is a quasi-central element of finite order in $\mathbf{T}^{1}$.

Proof. By [DM94, 1.16] there exists a quasi-central element $\sigma_{0}$ in $\mathbf{T}^{1}$. By Lemma 1.15 there exists $\sigma \in \mathbf{T}^{1}$ of finite order such that $C_{\mathbf{G}}(\sigma)=C_{\mathbf{G}}\left(\sigma_{0}\right)$, which implies by definition that $\sigma$ is quasi-central.

Context 1.23. In the rest of the text, we fix a quasi-central element $\sigma \in \mathbf{T}^{1}$ of finite order and $t$ will denote a finite order element of $\left(\mathbf{T}^{\sigma}\right)^{0}$.

Thus arbitrary quasi-semisimple elements (which may be chosen in $\left(\mathbf{T}^{\sigma}\right)^{0} \cdot \sigma$ by Proposition 1.16(1)) will now be denoted by $t \sigma$ — instead of $\sigma$ as they were before.

We have

$$
C_{t \sigma, \mathcal{O}(\alpha)} / C_{\sigma, \mathcal{O}(\alpha)}=\prod_{\beta \in \mathcal{O}(\alpha)} \beta(t)=\bar{\alpha}(t)
$$

where $\bar{\alpha}:=\sum_{\beta \in \mathcal{O}(\alpha)} \alpha$ and since $t \in\left(\mathbf{T}^{\sigma}\right)^{0}$, we have $\bar{\alpha}(t)=(|\mathcal{O}(\alpha)| \alpha)(t)$. 
Notation 1.25. By DM94, remarks following 1.22], when $\mathbf{G}$ is quasi-simple there is precisely one conjugacy class of quasi-central automorphisms inducing a given diagram automorphism of $W$, except for groups of type $A_{2 r}$ when $p \neq 2$, in which case there are two classes inducing the non-trivial diagram automorphism of $W$. For one of them $\left(\mathbf{G}^{\sigma}\right)^{0}$ is of type $B_{r}$ - this class disappears if $p=2-$ and for the other of type $C_{r}$. In the first case we say that $\sigma$ is of orthogonal type and in the second case of symplectic type (of course there can be several classes of elements inducing the same class of automorphisms, see Corollary 2.8).

Proposition 1.26. The simple roots of $\Sigma_{\sigma}$ corresponding to $\Sigma_{\sigma}^{+}$are the $\{\pi(\alpha) \mid$ $\alpha \in \Pi\}$, except that if $\mathcal{O}(\alpha)$ is special and $\pi(\alpha) \notin \Sigma_{\sigma}$, then one has to replace $\pi(\alpha)$ with $2 \pi(\alpha)$.

Proof. Using [St68, 1.32] we get that the basis of $\Sigma_{\sigma}$ corresponding to $\Sigma_{\sigma}^{+}$consists of those vectors colinear to the vectors of $\pi(\Pi)$ which are in $\Sigma_{\sigma}^{+}$. This gives the statement.

Remark 1.27. Note that, if there are no special orbits (in particular if $\Sigma$ has no component of type $A_{2 r}$ ), Definition 1.10 and Proposition 1.11 simplify substantially. We have just $\Sigma_{\sigma}=\{\pi(\alpha) \mid \alpha \in \Sigma\}$, and $\Sigma_{t \sigma}$ is a subsystem of $\Sigma_{\sigma}$, defined by the additional condition $\bar{\alpha}(t)=1$.

In type $A_{2 r}$, it may happen that $\Sigma_{t \sigma}$ is not a subsystem of $\Sigma_{\sigma}$ for any choice of $\sigma$. For instance, in $\mathbf{G}=\mathrm{GL}_{5}$, choose $\sigma$ as in [DM94, page 357], such that $\left(\mathbf{G}^{\sigma}\right)^{0} \simeq \mathrm{Sp}_{4}$ and preserving the diagonal torus. Number the simple roots $\alpha_{1}, \ldots, \alpha_{4}$ such that $\alpha_{i}(s)=\lambda_{i} / \lambda_{i+1}$ for $s=\operatorname{diag}\left(\lambda_{1}, \ldots, \lambda_{5}\right)$. For $t=\operatorname{diag}(i, 1,1,1,-i) \in\left(\mathbf{G}^{\sigma}\right)^{0}$, we get that $\Sigma_{t \sigma}^{+}=\left\{\pi\left(\alpha_{2}+\alpha_{3}\right), \pi\left(\alpha_{1}+\alpha_{2}\right)\right\}$ while $\Sigma_{\sigma}^{+}=\left\{\pi\left(\alpha_{1}\right), \pi\left(\alpha_{2}+\alpha_{3}\right), \pi\left(\alpha_{1}+\right.\right.$ $\left.\left.\alpha_{2}+\alpha_{3}+\alpha_{4}\right), \pi\left(\alpha_{1}+\alpha_{2}+\alpha_{3}\right)\right\}$, and for the representative $\sigma^{\prime}$ of the other class of quasi-central automorphisms whose fixed points are $\mathbf{G}^{\sigma^{\prime}}=\mathrm{O}_{5}$, we have $\Sigma_{\sigma^{\prime}}^{+}=$ $\left\{\pi\left(\alpha_{1}\right), \pi\left(\alpha_{2}\right), \pi\left(\alpha_{1}+\alpha_{2}\right), \pi\left(\alpha_{1}+\alpha_{2}+\alpha_{3}\right)\right\}$. Thus $\Sigma_{t \sigma}$ is not a subset of $\Sigma_{\sigma}$ or $\Sigma_{\sigma^{\prime}}$.

Proposition 1.28. We have $Z^{0}\left(\left(\mathbf{G}^{\sigma}\right)^{0}\right)=\left(Z(\mathbf{G})^{\sigma}\right)^{0}$, and the following are equivalent:

(1) $\mathbf{G}^{\sigma}$ is semisimple.

(2) $Y^{\sigma} \otimes \mathbb{Q}=\mathbb{Q} \Sigma_{\sigma}^{\vee}$.

(3) $\left(Z(\mathbf{G})^{\sigma}\right)^{0}=1$.

Proof. The first statement follows from [DM94, Corollaire 1.25(ii)] which applied for $\mathbf{L}=\mathbf{G}$ states that $C_{\mathbf{G}}\left(\operatorname{Rad}\left(\mathbf{G}^{\sigma}\right)^{0}\right)=\mathbf{G}$, thus $Z^{0}\left(\left(\mathbf{G}^{\sigma}\right)^{0}\right) \subset\left(Z(\mathbf{G})^{\sigma}\right)^{0}$. The converse inclusion is obvious.

The equivalence of items (1) to (3) is an immediate consequence of the first statement and the definitions.

Remark 1.29. If we do note take the identity component, it is not always true that $Z\left(\left(\mathbf{G}^{\sigma}\right)^{0}\right) \subset Z(\mathbf{G})$. A counterexample is in $\mathrm{GL}_{3}$ with $\sigma(x)=J^{t} x^{-1} J^{-1}$ where $J=\left(\begin{array}{ccc}0 & 0 & -1 \\ 0 & -1 & 0 \\ 1 & 0 & 0\end{array}\right)$. In that case $\left(\begin{array}{ccc}-1 & 0 & 0 \\ 0 & 1 & 0 \\ 0 & 0 & -1\end{array}\right) \in Z\left(\left(\mathbf{G}^{\sigma}\right)^{0}\right)$.

Note however that when $\sigma^{\prime}$ is quasi-central and there is no component of type $A_{2 r}$ where $\sigma^{\prime}$ is of symplectic type, then $Z\left(\mathbf{G}^{\sigma^{\prime} 0}\right) \subset Z(\mathbf{G})$. Indeed, then the root subgroups of $\mathbf{G}^{\sigma^{\prime} 0}$ involve all the root subgroups of $\mathbf{G}$, thus all roots of $\mathbf{G}$ must vanish on a central element in $\mathbf{G}^{\sigma^{\prime} 0}$. 
We now describe the centralizers of quasi-central elements for $\mathbf{G}$ quasi-simple.

Proposition 1.30. Assume that $\mathbf{G}$ is quasi-simple. Then $\left(\mathbf{G}^{\sigma}\right)^{0}$ is semisimple, and

(1) If $\mathbf{G}$ is adjoint not of type $A_{2 r}$ then $\mathbf{G}^{\sigma}=\left(\mathbf{G}^{\sigma}\right)^{0}$ is adjoint.

(2) If $\mathbf{G}$ is simply connected not of type $A_{2 r}$ then $\mathbf{G}^{\sigma}=\left(\mathbf{G}^{\sigma}\right)^{0}$ is simply connected.

(3) When $\mathbf{G}$ is of type $D_{4}$ and $n_{\sigma}=3$, then $\mathbf{G}$ is adjoint or simply connected and $\left(\mathbf{G}^{\sigma}\right)^{0}$ is of type $G_{2}$.

(4) When $\mathbf{G}$ is of type $D_{r}$ and $n_{\sigma}=2$, then $\mathbf{G}$ is adjoint or simply connected or equal to $\mathrm{SO}_{2 r}$. In this last case $\left(\mathbf{G}^{\sigma}\right)^{0}$ is adjoint of type $B_{r-1}$ and $\mathbf{G}^{\sigma}= \pm 1 \cdot\left(\mathbf{G}^{\sigma}\right)^{0}$.

(5) When $\mathbf{G}$ is of type $A_{2 r}$ then if $\sigma$ is of symplectic type then $\mathbf{G}^{\sigma}=\left(\mathbf{G}^{\sigma}\right)^{0}$ is simply connected of type $C_{r}$, and if $\sigma$ is of orthogonal type then $\mathbf{G}^{\sigma}=\left(\mathbf{G}^{\sigma}\right)^{0}$ is adjoint of type $B_{r}$.

(6) When $\mathbf{G}$ is of type $A_{2 r-1}$ and $n_{\sigma}=2$ then $\left(\mathbf{G}^{\sigma}\right)^{0}$ is of type $C_{r}$ and

- if $d=|P / X|$ is even, where $P$ is the weight lattice of $\Sigma$, then $\left(\mathbf{G}^{\sigma}\right)^{0}$ is adjoint and $\mathbf{G}^{\sigma}=\left(\mathbf{G}^{\sigma}\right)^{0}$ except if d divides $r$ and chark $\neq 2$ in which case $\mathbf{G}^{\sigma}= \pm 1 \cdot\left(\mathbf{G}^{\sigma}\right)^{0}$, where " -1 " is the image in $\mathbf{G}$ of a primitive $2 d$-th root of unity in the center of $\mathrm{SL}_{2 r}$.

- if $d=|P / X|$ is odd, $\mathbf{G}^{\sigma}=\left(\mathbf{G}^{\sigma}\right)^{0}$ is simply connected.

Proof. That $\mathbf{G}$ semisimple implies $\left(\mathbf{G}^{\sigma}\right)^{0}$ semisimple is a consequence of Proposition $1.28(3)$.

Observe that since $\sigma$ is quasi-central, proving that $\mathbf{G}^{\sigma}=\left(\mathbf{G}^{\sigma}\right)^{0}$ is equivalent to proving that $\mathbf{T}^{\sigma}=\left(\mathbf{T}^{\sigma}\right)^{0}$; this results from Proposition 1.12, the exact sequence of Proposition 1.14 and the fact that $W(\sigma)=W^{0}(\sigma)$ by Proposition 1.20(2). In particular, if $\mathbf{G}$ is adjoint or simply connected, then $\mathbf{G}^{\sigma}=\left(\mathbf{G}^{\sigma}\right)^{0}$ since in these cases we have $\mathbf{T}^{\sigma}=\left(\mathbf{T}^{\sigma}\right)^{0}$ by Lemma $1.2(2)$.

By Lemma 1.1 we have $X\left(\left(\mathbf{T}^{\sigma}\right)^{0}\right)=X_{\sigma}$. The weight lattice $P\left(\Sigma_{\sigma}\right)$ of $\Sigma_{\sigma}$ is the lattice dual to $\Sigma_{\sigma}^{\vee}$. If there is no special orbit $\Sigma_{\sigma}^{\vee}$ has basis $\left\{\bar{\alpha}^{\vee} \mid \alpha \in \Pi\right\}$, hence $P\left(\Sigma_{\sigma}\right)=\pi(P)$ where $P$ is the weight lattice of $\Sigma$. Thus if $P=X$ then $P\left(\Sigma_{\sigma}\right)=X\left(\left(\mathbf{T}^{\sigma}\right)^{0}\right)$ which shows $(2)$.

If there is no special orbit, since $\Sigma_{\sigma}=\pi(\Sigma)$, we get that the root lattice $Q\left(\Sigma_{\sigma}\right)$ of $\Sigma_{\sigma}$ is $\pi(Q)$ where $Q$ is the root lattice of $\Sigma$. Thus if $Q=X$ then $Q\left(\Sigma_{\sigma}\right)=$ $\pi(X)=X\left(\left(\mathbf{T}^{\sigma}\right)^{0}\right)$ which shows $(1)$.

If $\mathbf{G}$ is of type $D_{4}$, then with the notations of [Bbk, Planche IV] the fundamental weights are given in term of the simple roots by $\varpi_{1}=\alpha_{1}+\alpha_{2}+\alpha_{3} / 2+\alpha_{4} / 2, \varpi_{2}=$ $\alpha_{1}+2 \alpha_{2}+\alpha_{3}+\alpha_{4}, \varpi_{3}=\alpha_{1} / 2+\alpha_{2}+\alpha_{3}+\alpha_{4} / 2, \varpi_{4}=\alpha_{1} / 2+\alpha_{2}+\alpha_{3} / 2+\alpha_{4}$. Thus modulo $Q$ we have $2 \varpi_{1}=2 \varpi_{3}=2 \varpi_{4}=\varpi_{2}=0$ and $\varpi_{1}=\varpi_{3}+\varpi_{4}$. Hence we may take $\varpi_{3}, \varpi_{4}$ as a basis of $P / Q \simeq(\mathbb{Z} / 2 \mathbb{Z})^{2}$. The automorphism $\sigma$ of order $n_{\sigma}=3$ of $\Sigma$ is given by the permutation $\varpi_{1} \mapsto \varpi_{3} \mapsto \varpi_{4} \mapsto \varpi_{1}$. Its matrix in the basis $\varpi_{3}, \varpi_{4}$ of $P / Q$ is $\left(\begin{array}{ll}0 & 1 \\ 1 & 1\end{array}\right)$, which permutes circularly the three subgroups of order 2 of $P / Q$. Thus no lattice intermediate between $P$ and $Q$ is $\sigma$-stable, which implies that only the simply connected and adjoint group have an algebraic automorphism "triality" corresponding to $\sigma$.

When $\mathbf{G}$ is of type $D_{r}$ and $n_{\sigma}=2$, then $\left(\mathbf{G}^{\sigma}\right)^{0}$ is of type $B_{r-1}$ so that $\left|P\left(\Sigma_{\sigma}\right) / Q\left(\Sigma_{\sigma}\right)\right|=|\pi(P) / \pi(Q)|=2$. If $r$ is odd $P / Q \simeq \mathbb{Z} / 4 \mathbb{Z}$ and there is only one intermediate lattice $Q \subsetneq X \subsetneq P$ which corresponds to $\mathrm{SO}_{2 r}$. If $r$ is even then 
$P / Q \simeq(\mathbb{Z} / 2 \mathbb{Z})^{2}$ and there are 3 possible intermediate lattices. In this case the action of $\sigma$ fixes the lattice corresponding to $\mathrm{SO}_{2 r}$ and permutes the two others (see [D84, page 31]). This gives the first assertion of (4). If $\mathbf{G}=\mathrm{SO}_{2 r}$, let $\varpi_{1}, \ldots, \varpi_{r}$ be the fundamental weights, with $\sigma$ exchanging $\varpi_{r-1}$ and $\varpi_{r}$ (see Bbk, Planche IV, p. 256-257]); then $X$ is spanned by $\left\{\varpi_{1}, \ldots, \varpi_{r-2}, \varpi_{r-1}-\varpi_{r}, \varpi_{r-1}+\varpi_{r}\right\}$. Thus $\frac{1}{2}\left(\varpi_{r-1}+\varpi_{r}\right) \in \pi(P)-X_{\sigma}$ so that $X_{\sigma} \neq \pi(P)$. Since $|\pi(P) / \pi(Q)|=2$ we get $X_{\sigma}=\pi(Q)$ and $\left(\mathbf{G}^{\sigma}\right)^{0}$ is adjoint. The image $(\sigma-1) X$ is spanned by $2\left(\varpi_{r-1}-\varpi_{r}\right)$ while the kernel of $1+\sigma$ is spanned by $\varpi_{r-1}-\varpi_{r}$, thus according to Lemma[1.2(1) we have $\left|\mathbf{T}^{\sigma} /\left(\mathbf{T}^{\sigma}\right)^{0}\right|=2$. A representative is $-1 \notin\left(\mathbf{T}^{\sigma}\right)^{0}$, whence (4).

Assume now that $\mathbf{G}$ is of type $A_{r}$. Then the fundamental group $P\left(\Sigma_{\sigma}\right) / Q\left(\Sigma_{\sigma}\right)$ has order 2. Hence to show that $\left(\mathbf{G}^{\sigma}\right)^{0}$ is simply connected (resp. adjoint) it is sufficient to show that $X\left(\left(\mathbf{T}^{\sigma}\right)^{0}\right)$ is different from $Q\left(\Sigma_{\sigma}\right)$ (resp. from $P\left(\Sigma_{\sigma}\right)$ ).

Assume first that $\mathbf{G}$ is of type $A_{2 r}$ and that the orbit of $\alpha$ is special. If $\sigma$ is of symplectic type then $\pi(\alpha)$ is not in $Q\left(\Sigma_{\sigma}\right)$ but is in $\pi(Q) \subset X\left(\left(\mathbf{T}^{\sigma}\right)^{0}\right)$. Hence $\left(\mathbf{G}^{\sigma}\right)^{0}$ is simply connected. If $\sigma$ is of orthogonal type, then $\bar{\alpha}^{\vee}$ is not in the coroot lattice $Q\left(\Sigma_{\sigma}^{\vee}\right)$ hence $P\left(\Sigma_{\sigma}\right)$ is strictly larger than the dual of the lattice spanned by the $\bar{\alpha}^{\vee}$ which contains $X_{\sigma}=X\left(\left(\mathbf{T}^{\sigma}\right)^{0}\right)$, hence $\left(\mathbf{G}^{\sigma}\right)^{0}$ is adjoint.

Assume now that $\mathbf{G}$ is of type $A_{2 r-1}$. If $|P / X|=d$, then with the notations of Bbk, Planche I], $X$ is generated by $\alpha_{1}, \ldots, \alpha_{2 r-1}, d \varpi_{2 r-1}$, thus $X_{\sigma}$ is generated by $\pi(Q)$ and $\pi\left(d \varpi_{2 r-1}\right)$. Thus $X_{\sigma}=\pi(Q)$ if and only if $\pi\left(d \varpi_{2 r-1}\right)=\frac{d}{2}\left(\alpha_{1}+\ldots+\right.$ $\left.\alpha_{2 r-1}\right) \in \pi(Q)$, whence the result since $\frac{d}{2}\left(\alpha_{1}+\ldots+\alpha_{2 r-1}\right) \equiv \frac{d}{2} \alpha_{r}(\bmod \pi(Q))$.

We prove finally the assertions on $\mathbf{G}^{\sigma}$ in cases (5) and (6). Let $\mathbf{G}$ be of type $A_{l}$ and let $d=|P / X|$, a divisor of $l+1$. We keep the notation from Bbk Planche $\mathrm{I}]$ : the lattice $X$ is spanned by $Q$ and $d \varpi_{l}$. We want to use Lemma 1.2(1). For computing $\operatorname{Ker}(\sigma+1)$ and $(\sigma-1) X$ we introduce a suitable basis of $X$. Let $i_{1}:=2\left(\alpha_{l-1}-\alpha_{2}\right)+3\left(\alpha_{l-2}-\alpha_{3}\right)+\cdots+\left\lfloor\frac{l}{2}\right\rfloor\left(\alpha_{\left\lfloor\frac{l+3}{2}\right\rfloor}-\alpha_{\left\lfloor\frac{l}{2}\right\rfloor}\right)$ and $i_{0}:=\left(\alpha_{l}-\alpha_{1}\right)+i_{1}$. We use the notation $\lambda:=\left\{\begin{array}{ll}0 & \text { if } l \text { is even } \\ \frac{d}{2} \alpha_{(l+1) / 2} & \text { if } l \text { is odd }\end{array}\right.$ and $\mu:=-\frac{d}{l+1} i_{0}+\lambda$; then we claim that $\left\{\alpha_{2}, \alpha_{3}, \ldots, \alpha_{l}, \mu\right\}$ is a basis of $X$. Indeed $X$ has rank $l$, spanned by $\left\{\alpha_{1}, \ldots, \alpha_{l}, d \varpi_{l}\right\}$ and we have $\alpha_{1}=\frac{l+1}{d}(\mu-\lambda)+i_{1}+\alpha_{l}$ and $d \varpi_{l}=\frac{d}{l+1}\left(\alpha_{1}+\right.$ $\left.2 \alpha_{2}+3 \alpha_{3}+\cdots+l \alpha_{l}\right)=\mu+d\left(\alpha_{\left\lfloor\frac{l+3}{2}\right\rfloor}+\ldots+\alpha_{l}\right)$ so that $\mu \equiv d \varpi_{l}(\bmod Q)$. We have $\sigma\left(i_{0}\right)=-i_{0}$ and $\sigma(\lambda)=\lambda$ so that $\sigma(\mu)=\mu+\frac{2 d}{l+1} i_{0}$. We deduce that $(\sigma-1) X$ is spanned by $\left\{\alpha_{l}-\alpha_{1}, \alpha_{l-1}-\alpha_{2}, \ldots, \alpha_{\left\lfloor\frac{l+3}{2}\right\rfloor}-\alpha_{\left\lfloor\frac{l}{2}\right\rfloor}, \frac{2 d}{l+1} i_{0}\right\}$ that is by $\left\{\alpha_{l-1}-\alpha_{2}, \ldots, i_{0}, \frac{2 d}{l+1} i_{0}\right\}$, since $\alpha_{l}-\alpha_{1}=i_{0}-i_{1}$ and $i_{1}$ is an integral combination of $\left\{\alpha_{l-1}-\alpha_{2}, \ldots\right\}$.

We compute now the kernel of $1+\sigma$. We have $(1+\sigma)\left(a_{2} \alpha_{2}+\cdots+a_{l} \alpha_{l}+b \mu\right)=$ $\left.\left(a_{2}+a_{l-1}\right)\left(\alpha_{2}+\alpha_{l-1}\right)+\left(a_{3}+a_{l-2}\right)\left(\alpha_{3}+\alpha_{l-2}\right)+\cdots+a_{l}\left(\alpha_{l}+\alpha_{1}\right)+2 b \lambda\right\}$. Since in this sum $\alpha_{l}+\alpha_{1}$ is the only term with a non-zero coefficient on $\mu$ when written in the above basis of $X$, the elements of the kernel of $1+\sigma$ have $a_{l}=0, a_{i}=-a_{l+1-i}$ for $i=2, \ldots,\left\lfloor\frac{l}{2}\right\rfloor$ and, if $l$ is odd $d b=2 a_{\frac{l+1}{2}}$ (otherwise $b$ is arbitrary). We get that $\operatorname{ker}(\sigma+1)$ is spanned by the $\alpha_{i}-\alpha_{l+1-i}$ for $i=2, \ldots,\left\lfloor\frac{l}{2}\right\rfloor$ and $\mu-\lambda$ if $\mu-\lambda \in X$ otherwise by the $\alpha_{i}-\alpha_{l+1-i}$ and $2(\mu-\lambda)$. Since $\mu-\lambda$ is in $X$ if and only if $\lambda=0$ or $l$ is odd, we get $\operatorname{ker}(1+\sigma)=\left\langle\left(\alpha_{i}-\alpha_{l+1-i}\right)_{i=2, \ldots,\left\lfloor\frac{l}{2}\right\rfloor}, \frac{d}{l+1} i_{0}\right\rangle$ if $l$ is even or $d$ is even, and $\operatorname{ker}(1+\sigma)=\left\langle\left(\alpha_{i}-\alpha_{l+1-i}\right)_{i=2, \ldots,\left\lfloor\frac{l}{2}\right\rfloor}, \frac{2 d}{l+1} i_{0}\right\rangle$ if $l$ and $d$ are odd. Hence $\operatorname{ker}(1+\sigma) /(\sigma-1) X$ is non-trivial (of order 2) only if $l$ is even or $d$ is even and $\frac{d}{l+1} i_{0}$ is not in the span of $\left\langle i_{0}, \frac{2 d}{l+1} i_{0}\right\rangle$ thus if and only if $d$ is even and $2 d$ divides 
$l+1$. Writing $l=2 r-1$ in that case, we get $2 d=2|P / X|$ divides $2 r$ that is $|P / X|$ divides $r$.

\section{The root Datum $R(\sigma)$ and its affine Weyl group}

We recall that by Context 1.23, $\sigma$ denotes a quasi-central element (a more general quasi-semisimple element is denoted by $t \sigma$ ).

Context 2.1. From now on, when there is a component $\mathbf{H}$ of $\mathbf{G}$ of type $A_{2 r}$ and $p \neq 2$, we assume that the smallest power $\sigma^{i}$ which stabilizes that component is such that $\mathbf{H}^{\sigma^{i}}$ is of type $A_{2 r}$ or $C_{r}$ (and not of type $B_{r}$ ), that is on that component $\sigma^{i}$ acts trivially or is of symplectic type.

Note that when $p=2$ there is no choice to make: $\mathbf{H}^{\sigma^{i}}$ is of type $A_{2 r}$ if $\sigma^{i}$ acts trivially on $\mathbf{H}$ and of type $C_{r}$ otherwise. Context 2.1 chooses in every case a well-defined conjugacy class of quasi-central automorphisms $\sigma$.

Proposition 2.2. It follows from the choice made in Context 2.1 that for $\alpha \in \Sigma$ we have $C_{\sigma, \mathcal{O}(\alpha)}=1$, unless $\mathcal{O}(\alpha)$ is special and $p \neq 2$, in which case $C_{\sigma, \mathcal{O}(\alpha)}=-1$.

Proof. This follows from [DM94, 1.8(v) and second example page 357] and from DM02, 2.5].

We now introduce a root datum $R(\sigma)=\left(\Phi_{\sigma}, \Phi_{\sigma}^{\vee}, X^{\sigma}, Y_{\sigma}\right)$, (recall from Lemma 1.6 that $X^{\sigma}=X(\mathbf{T} /[\mathbf{T}, \sigma])$ and $\left.Y_{\sigma}=Y(\mathbf{T} /[\mathbf{T}, \sigma])\right)$ which will have the advantage over $\Sigma_{\sigma}$ that the system $\Phi_{t \sigma}$ associated to $t \sigma \in\left(\mathbf{T}^{\sigma}\right)^{0} \cdot \sigma$ will be a closed subsystem of $\Phi_{\sigma}$, closely related to $\Sigma_{t \sigma}$. The root system introduced in [Sp06, Proposition 2] is equal to $\Phi_{\sigma}$ if there are no special orbits, but is not as well behaved when they exist.

\section{Proposition 2.3.}

$\Phi_{\sigma}:=\{\bar{\alpha} \mid \alpha \in \Sigma, \mathcal{O}(\alpha)$ not special or cospecial $\} \cup\{2 \bar{\alpha} \mid \alpha \in \Sigma, \mathcal{O}(\alpha)$ special $\} \subset X^{\sigma}$ and $\Phi_{\sigma}^{\vee}:=\left\{\pi\left(\alpha^{\vee}\right) \mid \alpha \in \Sigma, \mathcal{O}(\alpha)\right.$ is not cospecial $\} \subset Y_{\sigma}$, are the roots and the coroots of a reduced root system for $W^{\sigma}$ acting as in Proposition 1.16](2); the root corresponding to the coroot $\pi\left(\alpha^{\vee}\right)$ is $\bar{\alpha}$ or $2 \bar{\alpha}$.

The set $\Phi_{\sigma}^{+}:=\Phi_{\sigma} \cap \mathbb{Q}^{+} \Pi$ is a set of positive roots for $\Phi_{\sigma}$ and

$$
\Delta_{\sigma}:=\{\bar{\alpha} \mid \alpha \in \Pi, \mathcal{O}(\alpha) \text { not special }\} \cup\{2 \bar{\alpha} \mid \alpha \in \Pi, \mathcal{O}(\alpha) \text { special }\}
$$

is the corresponding set of simple roots. The weight lattice $P\left(\Phi_{\sigma}\right)$ is equal to $P^{\sigma}$ where $P$ is the weight lattice of $\Sigma$. The fundamental coweights of the root system $\left(\Phi_{\sigma}, \Phi_{\sigma}^{\vee}\right)$ are $\left\{\pi\left(\varpi_{\alpha}^{\vee}\right) \mid \alpha \in \Pi, \mathcal{O}(\alpha)\right.$ not special $\} \cup\left\{\frac{1}{2} \pi\left(\varpi_{\alpha}^{\vee}\right) \mid \alpha \in \Pi, \mathcal{O}(\alpha)\right.$ special $\}$, where $\left\{\varpi_{\alpha}^{\vee} \mid \alpha \in \Pi\right\}$ are the fundamental coweights of the root system $\Sigma$.

Proof. By [DM02, 2.3] and its proof the set $\Phi_{\sigma}^{\prime}$ obtained from $\Phi_{\sigma}$ by replacing $2 \bar{\alpha}$ with $\bar{\alpha}$ for special orbits, is a reduced root system in $X^{\sigma} \otimes \mathbb{Q}$ for the group $W^{\sigma}$. Since $W^{\sigma}$ maps a special orbit to a special orbit, it stabilizes $\Phi_{\sigma}$. Since the vectors in $\Phi_{\sigma}$ are colinear to the vectors in $\Phi_{\sigma}^{\prime}$, the reflections of $W^{\sigma}$ are reflections with respect to the elements of $\Phi_{\sigma}$. To prove that $\Phi_{\sigma}$ is a root system and $\Phi_{\sigma}^{\vee}$ is the corresponding set of coroots it suffices to prove that the pairing between $X^{\sigma} \otimes \mathbb{Q}$ and $Y_{\sigma} \otimes \mathbb{Q}$ takes integral values on $\Phi_{\sigma} \times \Phi_{\sigma}^{\vee}$ with diagonal values equal to 2 .

For all roots $\alpha$ and $\beta$ we have $\left(\pi\left(\beta^{\vee}\right), \bar{\alpha}\right)=\frac{1}{|\mathcal{O}(\beta)|} \sum_{\gamma \in \mathcal{O}(\beta)}\left(\gamma^{\vee}, \bar{\alpha}\right)$. Since the pairing is $\sigma$-invariant, this is equal to $\left(\beta^{\vee}, \bar{\alpha}\right)$ which is an integer. In particular 
$\left(\pi\left(\alpha^{\vee}\right), \bar{\alpha}\right)=\left(\alpha^{\vee}, \bar{\alpha}\right)$; by Remark 1.9 this is equal to 2 if $\alpha$ is not special and to 1 if $\alpha$ is special, so that in the latter case $\left(\pi\left(\alpha^{\vee}\right), 2 \bar{\alpha}\right)=2$. We have proved the assertions on $\Phi_{\sigma}$ and $\Phi_{\sigma}^{\vee}$.

The assertions on the positive and simple roots result from the fact that the root $\bar{\alpha}$ is a positive multiple of $\pi(\alpha)$.

To prove the assertion on the weights, first notice that the fundamental weights $\left\{\varpi_{\alpha} \mid \alpha \in \Pi\right\}$ are permuted by $\sigma$ since they are the dual basis to the simple coroots. Thus $\left\{\sum_{\beta \in \mathcal{O}} \varpi_{\beta}\right\}_{\mathcal{O} \in \Pi / \sigma}$ is a basis of $P^{\sigma}$. Now for all simple roots $\alpha$ and $\gamma$

$$
\sum_{\beta \in \mathcal{O}(\alpha)} \varpi_{\beta}\left(\pi\left(\gamma^{\vee}\right)\right)= \begin{cases}1 & \text { if } \gamma \in \mathcal{O}(\alpha) \text { and } \mathcal{O}(\alpha) \text { is not cospecial, } \\ 0 & \text { otherwise. }\end{cases}
$$

This shows that $P^{\sigma}$ is indeed dual to $\mathbb{Z} \Phi_{\sigma}^{\vee}$.

The assertion on coweights comes from a similar computation: for $\alpha, \beta \in \Pi$ we have $\left(\pi\left(\varpi_{\alpha}^{\vee}\right), \bar{\beta}\right)=\left(\varpi_{\alpha}^{\vee}, \bar{\beta}\right)=\delta_{\mathcal{O}(\alpha), \mathcal{O}(\beta)}$, by invariance of the pairing. If $\mathcal{O}(\alpha)$ is not special then $\bar{\alpha} \in \Delta_{\sigma}$ and the corresponding fundamental coweight is $\pi\left(\varpi_{\alpha}^{\vee}\right)$; if $\mathcal{O}(\alpha)$ is special then $2 \bar{\alpha} \in \Delta_{\sigma}$ and the corresponding fundamental coweight is $\frac{1}{2} \pi\left(\varpi_{\alpha}^{\vee}\right)$

The following proposition is, for the root datum $R(\sigma)$, the analogue of Proposition 1.30 for the root datum $\left(\Sigma_{\sigma}, \Sigma_{\sigma}^{\vee}, X_{\sigma}, Y^{\sigma}\right)$

Proposition 2.4. Let $R(\sigma)$ be the root datum $\left(\Phi_{\sigma}, \Phi_{\sigma}^{\vee}, X^{\sigma}, Y_{\sigma}\right)$. Assume that $\mathbf{G}$ is quasi-simple; then $Y_{\sigma} \otimes \mathbb{Q}=\mathbb{Q} \Phi_{\sigma}^{\vee}$, that is $R(\sigma)$ is semisimple, and

(1) If $\mathbf{G}$ is adjoint not of type $A_{2 r}$ then $R(\sigma)$ is of adjoint type.

(2) If $\mathbf{G}$ is simply connected then $R(\sigma)$ is of simply connected type.

(3) If $\mathbf{G}=\mathrm{SO}_{2 r}$ and $n_{\sigma}=2$, then $R(\sigma)$ is of type $C_{r-1}$ simply connected.

(4) If $\mathbf{G}$ is of type $A_{2 r}$ and $n_{\sigma}=2$ then $R(\sigma)$ is of type $C_{r}$ simply connected.

(5) When $\mathbf{G}$ is of type $A_{2 r-1}$ and $n_{\sigma}=2$ then if $\left|P^{\vee} / Y\right|$ is even, where $P^{\vee}$ is the coweight lattice of $\Sigma$, then $R(\sigma)$ is of type $B_{r}$ simply connected; otherwise $R(\sigma)$ is of type $B_{r}$ adjoint.

Proof. That G semisimple implies $R(\sigma)$ semisimple is an immediate consequence of Proposition $1.28(2)$ since $Y_{\sigma} \otimes \mathbb{Q}=\mathbb{Q} \Phi_{\sigma}^{\vee}$ is clearly equivalent to $Y^{\sigma} \otimes \mathbb{Q}=\mathbb{Q} \Sigma_{\sigma}^{\vee}$.

Recall that $P$ and $Q$ denote the weight and root lattice of $\Sigma$. We denote by $P^{\vee}$ and $Q^{\vee}$ the coweight and coroot lattice of $\Sigma$.

If there is no special orbit the root lattice $Q\left(\Phi_{\sigma}\right)$ of $\Phi_{\sigma}$ is equal to $Q^{\sigma}$. Hence, if $X=Q$ then $X^{\sigma}=Q\left(\Phi_{\sigma}\right)$, whence (1).

Similarly, if $X=P$ then $X^{\sigma}=P^{\sigma}=P\left(\Phi_{\sigma}\right)$, the last equality by Proposition 2.3. whence (2) for type not $A_{2 r}$. For this last type, see the proof of (4).

If $\mathbf{G}=\mathrm{SO}_{2 r}$ we use the same computation as in the proof of Proposition 1.30(4), replacing weights with coweights and $X$ with $Y$. This gives $Y_{\sigma} \neq \pi\left(P^{\vee}\right)$ so that $Y_{\sigma}=\pi\left(Q^{\vee}\right)$ since $\left|\pi\left(P^{\vee}\right) / \pi\left(Q^{\vee}\right)\right|=2$, whence (3) since $\pi\left(Q^{\vee}\right)$ is the coroot lattice of $\Phi_{\sigma}$. There is one orbit of simple roots with 2 elements, which gives a long simple root in $\Phi_{\sigma}$ so that the type of $R(\sigma)$ is $C_{r-1}$.

If $\mathbf{G}$ is of type $A_{r}$ we have $\left|P\left(\Phi_{\sigma}^{\vee}\right) / Q\left(\Phi_{\sigma}^{\vee}\right)\right|=2$. Hence to show that $R(\sigma)$ is of adjoint type (resp. of simply connected type) it is sufficient to show that $Y_{\sigma}$ is different from $Q\left(\Phi_{\sigma}^{\vee}\right)=\pi\left(Q^{\vee}\right)$ (resp. from $P\left(\Phi_{\sigma}^{\vee}\right)=\pi\left(P^{\vee}\right)$ ).

If $\mathbf{G}$ is of type $A_{2 r}$ and $n_{\sigma}=2$, then for $\alpha \in \Pi$ such that $\mathcal{O}(\alpha)$ is special, $\frac{1}{2} \pi\left(\varpi_{\alpha}^{\vee}\right)=\frac{1}{4}\left(\varpi_{\alpha}+\varpi_{\sigma(\alpha)}\right)$ is a coweight of $\Phi_{\sigma}$ but is not in $\pi\left(P^{\vee}\right)$, hence is not in 
$Y_{\sigma}=\pi(Y)$. Thus $Y_{\sigma} \neq \pi\left(P^{\vee}\right)$, and $R(\sigma)$ is of simply connected type. There is one special orbit of simple roots which gives a long simple root in $\Phi_{\sigma}$ so that $R(\sigma)$ is of type $C_{r}$.

Assume $\mathbf{G}$ of type $A_{2 r-1}$ and $n_{\sigma}=2$. Since exchanging the roots and the coroots in type $A$ gives an isomorphic root system, we can make the same computation as in the proof of Proposition $1.30(6)$, exchanging $Q$ with $Q^{\vee}, P$ with $P^{\vee}$, and $X$ with $Y$. This gives that $Y_{\sigma}=\pi\left(Q^{\vee}\right)$ if and only if $\left|P^{\vee} / Y\right|$ is even, whence the result. There is no special orbit of simple roots and one orbit is a singleton, so that this orbit gives a short root in $\Phi_{\sigma}$ and the type is $B_{r}$.

We recall that $\left(W^{\sigma}, S_{\sigma}\right)$ is a Coxeter system (see Proposition $1.11(2)$ ).

Proposition 2.5. Let $W_{a}:=Q\left(\Phi_{\sigma}^{\vee}\right) \rtimes W^{\sigma}$ be the affine Weyl group where $Q\left(\Phi_{\sigma}^{\vee}\right)=$ $\mathbb{Z} \Phi_{\sigma}^{\vee}$ is the coroot lattice. Let us denote by $\left(W_{i}^{\sigma}\right)_{i \in I}$ the irreducible components of $W^{\sigma}$ and by $\Phi_{\sigma, i}$ (resp. $S_{\sigma, i}$ ) the corresponding subsets of $\Phi_{\sigma}$ (resp. $\left.S_{\sigma}\right)$. For $i \in I$, let $s_{0, i}$ be the reflection with respect to the hyperplane $\alpha_{0, i}(x)=1$, where $\alpha_{0, i}$ is the highest root of $\Phi_{\sigma, i}$ for the order $\Phi_{\sigma}^{+}$. Finally, let $\tilde{S}_{\sigma, i}:=S_{\sigma, i} \cup\left\{s_{0, i}\right\}$ and let $\tilde{S}_{\sigma}=\cup_{i} \tilde{S}_{\sigma, i}$.

(1) $\left(W_{a}, \tilde{S}_{\sigma}\right)$ is a Coxeter system.

(2) The closure $\mathcal{C}$ of the fundamental alcove of the hyperplane system of $W_{a}$ is a fundamental domain for $W_{a}$ acting on $\mathbb{Q} \Phi_{\sigma}^{\vee}$. It is the product of the simplices $\mathcal{C}_{i}$ with vertices 0 and $\left\{\frac{1}{n_{s}} \varpi_{s}^{\vee} \mid s \in S_{\sigma, i}\right\}$, where $\left\{\varpi_{s}^{\vee}\right\}_{s \in S_{\sigma}}$ are the fundamental coweights of the root system $\left(\Phi_{\sigma}, \Phi_{\sigma}^{\vee}\right)$ and $\sum_{s \in S_{\sigma, i}} n_{s} \alpha_{s}$ is the expression of $\alpha_{0, i}$ in term of the simple roots.

(3) $\tilde{S}_{\sigma}$ is the set of reflections with respect to the walls of $\mathcal{C}$.

(4) Let $\tilde{\Delta}_{\sigma}:=\Delta_{\sigma} \cup\left\{-\alpha_{0, i} \mid i \in I\right\}$. For each $i \in I$, let $J_{i}:=\left\{s \in S_{\sigma, i} \mid n_{s}=1\right\}$. and for $s \in J_{i}$ let $z_{s}:=w_{S_{\sigma, i}-\{s\}} w_{S_{\sigma, i}}$; then $z_{s}$ is an automorphism of $\tilde{\Delta}_{\sigma}$ and $\prod_{i \in I}\left(\left\{z_{s} \mid s \in J_{i}\right\} \cup\{\mathrm{Id}\}\right)$ is the group of all automorphisms of $\tilde{\Delta}_{\sigma}$ induced by $W^{\sigma}$.

(5) Let $W_{a}^{\prime}:=P\left(\Phi_{\sigma}^{\vee}\right) \rtimes W^{\sigma}$ be the semi-direct product by the coweight lattice, giving a natural isomorphism $j: W_{a}^{\prime} / W_{a} \stackrel{\sim}{\rightarrow} P\left(\Phi_{\sigma}^{\vee}\right) / Q\left(\Phi_{\sigma}^{\vee}\right)$. Then $\prod_{i \in I}\left(\{0\} \cup\left\{\varpi_{s}^{\vee} \mid s \in J_{i}\right\}\right)$ is a set of representatives of $P\left(\Phi_{\sigma}^{\vee}\right) / Q\left(\Phi_{\sigma}^{\vee}\right)$. For $s \in J_{i}$, let $\gamma_{s}$ be the composed of $z_{s}$ and the translation by $\varpi_{s}^{\vee}$; then $\gamma_{s}\left(\mathcal{C}_{i}\right)=\mathcal{C}_{i}$. Composing $j$ with the Cartesian product over $I$ of the maps which send $\varpi_{s}^{\vee}$ to $\gamma_{s}$ and 0 to Id we get a group isomorphism from $W_{a}^{\prime} / W_{a}$ to the automorphisms of $\mathcal{C}$ induced by $W_{a}^{\prime}$.

A consequence of (5) is that if $\mathbf{G}^{\sigma}$ is semisimple, $\widetilde{W}$ is generated by $W_{a}$ and some of the $\gamma_{s}$.

Note also that $\gamma_{s} \mapsto z_{s}$ is a bijection, thus (4) and (5) establish isomorphisms between the groups $W_{a}^{\prime} / W_{a}, \operatorname{Aut}_{W^{\sigma}}\left(\tilde{\Delta}_{\sigma}\right), \operatorname{Aut}_{W_{a}^{\prime}}(\mathcal{C})$ and $P\left(\Phi_{\sigma}^{\vee}\right) / Q\left(\Phi_{\sigma}^{\vee}\right)$.

Proof. Statement (1) comes from [Bbk, Ch. V, §3, Th.1] and Bbk, Ch. VI §2, Prop.5].

The fact that $\mathcal{C}$ is a fundamental domain, and its decomposition as a product of simplices is stated in [Bbk, Ch. VI, $\S 2$, end of no 1]. The formula for the vertices of the fundamental alcove of each irreducible component is given in $\mathrm{Bbk}, \mathrm{Ch}$. VI, $\S 2$ Cor. of Prop.5]

Statement (3) is Bbk, Ch. VI, $\S 2$ Prop.5 (i)]. 
Statement (4) is B05. (3.5)]

Statement (5) is deduced from [Bbk, Ch. VI, $\S 2$ no 3, Prop.6 and its corollary] which discusses the case of an irreducible Coxeter group.

One gets the consequence of (5) mentioned after the statement using that, as observed at the beginning of the proof of Proposition 2.4. if $\mathbf{G}^{\sigma}$ is semisimple then $R(\sigma)$ also, thus $\widetilde{W}$ is a subgroup of $W_{a}^{\prime}$.

Note that Proposition 2.5(5) allows when $\mathbf{G}^{\sigma}$ is semisimple to see the fundamental domain of $\widetilde{W}$ as a subset of $\mathcal{C}$.

With the convention of Context 2.1, we have:

Corollary 2.6. The G-conjugacy classes of quasi-semisimple elements of finite order in $\mathbf{G}^{1}$ are parameterized by the points of a fundamental domain of $\widetilde{W}$ in $Y_{\sigma} \otimes \mathbb{Q}_{p^{\prime}}$. If the class of t $\sigma$ is parameterized by $\lambda$, the root system $\Sigma_{t \sigma}$ of $\left(\mathbf{G}^{t \sigma}\right)^{0}$ is

$$
\left\{\begin{array}{l|l}
\pi(\alpha), \alpha \in \Sigma \text { such that } \mathcal{O}(\alpha) \text { is } & \begin{array}{l}
\text { special and } 2 \bar{\alpha}(\lambda) \in 2 \mathbb{Z}+1 \\
\text { not special and } \bar{\alpha}(\lambda) \in \mathbb{Z}
\end{array}
\end{array}\right\} \text {. }
$$

Note that the conditions above corresponds to $\lambda$ lying on one of the affine hyperplanes of $W_{a}$.

Proof. The first assertion is clear from Theorem 1.17. We get the second assertion by noticing that the condition which defines $\Sigma_{t \sigma}$ in Definition 1.10 can be written $\bar{\alpha}(\lambda) \in \mathbb{Z}$ when $C_{\sigma, \mathcal{O}(\alpha)}=1$. Similarly, when $C_{\sigma, \mathcal{O}(\alpha)}=-1$ the condition can be written $2 \bar{\alpha}(\lambda) \in 2 \mathbb{Z}+1$.

Proposition 2.7. $\Phi_{t \sigma}:=\left\{\alpha \in \Phi_{\sigma} \mid \alpha(t)=1\right\}$ is a closed subsystem of $\Phi_{\sigma}$, and is a root system for the reflection subgroup $W^{0}(t \sigma)$ of $W^{\sigma}$. In other terms, if $\lambda \in Y_{\sigma} \otimes \mathbb{Q}$ parameterizes the class of t $\sigma$ as in Corollary 2.6. we have $W^{0}(t \sigma)=\left\langle s_{\alpha} \in W^{\sigma}, \alpha \in\right.$ $\Phi_{\sigma}\left|s_{\alpha}(\lambda)-\lambda \in \mathbb{Z} \Phi_{\sigma}^{\vee}\right\rangle$.

In the above $\alpha(t)$ is the evaluation of $\alpha \in X^{\sigma}$ on $t$.

Proof. $\Phi_{t \sigma}$ is a closed subsystem of $\Phi_{\sigma}$ since the condition which defines it is clearly closed. Let us show that the roots of $\Phi_{t \sigma}$ and $\Sigma_{t \sigma}$ are colinear. If $\mathcal{O}(\alpha)$ is not special or cospecial then $\bar{\alpha}$ is a root of $\Phi_{\sigma}$ and by Equation 1.24 the condition $\bar{\alpha}(t)=1$ is equivalent to $\pi(\alpha) \in \Sigma_{t \sigma}$, thus $\bar{\alpha} \in \Phi_{t \sigma}$ if and only if $\pi(\alpha) \in \Sigma_{t \sigma}$. If $\mathcal{O}(\alpha)$ is special the corresponding root of $\Phi_{\sigma}$ is $2 \bar{\alpha}$ so the condition for $2 \bar{\alpha}$ to be in $\Phi_{t \sigma}$ is $\bar{\alpha}(t)= \pm 1$. Each of the signs determines a root in $\Sigma_{t \sigma}$ colinear to $\bar{\alpha}$ : with our choice of $\sigma$ (see Context 2.1) in case $A_{2 r}$ this root is $\pi(\alpha)$ if $\bar{\alpha}(t)=-1$, and $\pi\left(\alpha+\sigma^{i}(\alpha)\right)=2 \pi(\alpha)$ otherwise, where $2 i=|\mathcal{O}(\alpha)|$. Thus by [DM15, remark after 8.5], the root systems $\Phi_{t \sigma}$ and $\Sigma_{t \sigma}$ have same Weyl group, which is $W^{0}(t \sigma)$ by Proposition 1.11, Finally $s_{\alpha}(\lambda)-\lambda=-\alpha(\lambda) \alpha^{\vee}$ thus $\alpha(\lambda) \in \mathbb{Z}$ is equivalent to $s_{\alpha}(\lambda)-\lambda \in \mathbb{Z} \Phi_{\sigma}^{\vee}$.

Corollary 2.8. The $\mathbf{G}$-classes of quasi-central elements in $\mathbf{G}^{1}$ are in bijection with the center of $R(\sigma)$, where we define the center of the root datum $R(\sigma)$ as the set of orbits of $\widetilde{W} / W_{a}$ on $\prod_{i \in I}\left(\{0\} \cup\left\{\varpi_{s}^{\vee} \mid s \in J_{i}\right\}\right)$.

We note in particular that though for a quasi-simple group not of type $A_{2 n}$ there is a single class of quasi-central automorphisms by [DM94, 1.22], there is more than one class of quasi-central elements whenever the datum $R(\sigma)$ is not adjoint, which, as described in Proposition 2.4, often happens. 
Proof. The class of $t \sigma \in \mathbf{G}^{1}$ is quasi-central if and only if $W^{0}(t \sigma)=W^{\sigma}$, hence, by Proposition 2.7 if and only if $s_{\alpha}(\lambda)-\lambda \in \mathbb{Z} \Phi_{\sigma}^{\vee}$ for all $\alpha \in \Phi_{\sigma}$, where $\lambda \in \mathcal{C}$ parameterizes the class of $t \sigma$. This is equivalent to $\alpha(\lambda) \in \mathbb{Z}$, which is equivalent to the same condition for all $\alpha \in \Delta_{\sigma}$. Since $\lambda$ is in $\mathcal{C}$ we have $\lambda=\sum_{i \in I} \sum_{s \in S_{\sigma, i}} \frac{\lambda_{s}}{n_{s}} \varpi_{s}^{\vee}$ with $\lambda_{s} \geq 0$ and $\sum_{s \in S_{\sigma, i}} \lambda_{s}=1$ for any $i$. If $\alpha \in \Delta_{\sigma}$ corresponds to $s \in S_{\sigma}$ one has $\alpha(\lambda)=\frac{\lambda_{s}}{n_{s}} \in\left[0, \frac{1}{n_{s}}\right]$, hence $\alpha(\lambda) \in \mathbb{Z}$ if and only if $\lambda_{s}=0$ or $\lambda_{s}=n_{s}=1$. From the condition $\sum_{s \in S_{\sigma, i}} \lambda_{s}=1$ we deduce that for each $i$ there is at most one non-zero $\lambda_{s}$ with $s \in S_{\sigma, i}$, which means that $\lambda \in \prod_{i \in I}\left(\{0\} \cup\left\{\varpi_{s}^{\vee} \mid s \in J_{i}\right\}\right)$. By Corollary 2.6. the quasi-central classes are parameterized by the $\widetilde{W}$-orbits of such $\lambda$.

We call minuscule the weights of the form $\sum_{i \in K} \varpi_{s_{i}}$ where $K \subset I$ is non-empty and where, for each $i \in K$ the fundamental weight $\varpi_{s_{i}}$ corresponds to $s_{i} \in J_{i}$ (this extends the usual definition of minuscule weights in the irreducible case).

Lemma 2.9. Let $Q$ be the root lattice of $\Sigma$ in $X \otimes \mathbb{Q}$. Then the non-zero elements of $P^{\sigma} / Q^{\sigma}$ identify with certain $\sigma$-stable minuscule weights. If $\mathbf{G}$ is semisimple we have $Q \subset X \subset P$, thus if $\sigma$ fixes no minuscule weight, then $Q^{\sigma}=X^{\sigma}=P^{\sigma}$.

Proof. When $\Sigma$ is irreducible, a $\sigma$-stable set of representatives of the non-zero elements of $P / Q$ is given by the minuscule weights (see Bbk, VI, $\S 2$, exercice $5]$ ); thus in general such a set is given by the minuscule weights with our extended definition. The first assertion is thus a consequence of the exact sequence $0 \rightarrow Q^{\sigma} \rightarrow P^{\sigma} \rightarrow(P / Q)^{\sigma}$. The second assertion follows.

Example 2.10. Consider the case of $\mathbf{G}$ semisimple of type $D_{4}$ with the triality automorphism $\sigma$. There are two subcases, $\mathbf{G}$ simply connected or adjoint - the intermediate cases do not admit a triality, see Proposition 1.30(3).

- When $\mathbf{G}$ is adjoint, $X=Q$ has basis the simple roots $\alpha_{1}, \alpha_{2}, \alpha_{3}, \alpha_{4}$ and $\sigma$ is given by the cycle $\alpha_{1} \mapsto \alpha_{3} \mapsto \alpha_{4}$.

- When $\mathbf{G}$ is simply connected, $X=P$ has basis the weights $\varpi_{1}, \varpi_{2}, \varpi_{3}, \varpi_{4}$ and $\sigma$ is given by the cycle $\varpi_{1} \mapsto \varpi_{3} \mapsto \varpi_{4}$.

The minuscule weights are $\varpi_{1}, \varpi_{3}, \varpi_{4}$ and none is fixed by $\sigma$. By the second assertion of Lemma [2.9] it follows that if we set $\alpha:=\alpha_{1}+\alpha_{3}+\alpha_{4}$ and $\beta:=\alpha_{2}$ (the simple roots of the root system $G_{2}=\left(\Phi_{\sigma}, \Phi_{\sigma}^{\vee}\right)$ ) in both cases $X^{\sigma}=P^{\sigma}=Q^{\sigma}$ has basis $\alpha, \beta$. The highest root of the root system $G_{2}$ is $2 \alpha+3 \beta$, in both cases $Y_{\sigma}$ has basis the simple coroots of $G_{2}$ given by $\alpha^{\vee}=\left(\alpha_{1}^{\vee}+\alpha_{3}^{\vee}+\alpha_{4}^{\vee}\right) / 3$ and $\beta^{\vee}=\alpha_{2}^{\vee}$ and the fundamental coweights are $\varpi_{\alpha}^{\vee}=2 \alpha^{\vee}+\beta^{\vee}$ and $\varpi_{\beta}^{\vee}=3 \alpha^{\vee}+2 \beta^{\vee}$. We have $\widetilde{W}=W_{a}$ and the extremal points of the fundamental alcove are given by $0, \alpha^{\vee}+\frac{1}{2} \beta^{\vee}$ and $\alpha^{\vee}+\frac{2}{3} \beta^{\vee}$. By Corollary 2.6, the corresponding root systems of $\left(\mathbf{G}^{t \sigma}\right)^{0}$ are respectively of type $G_{2}, A_{1} \times A_{1}$ and $A_{2}$ - see also the table at the end of the paper.

The following remark will be useful

Proposition 2.11. When $\mathbf{G}$ is quasi-simple, $n_{\sigma}>1$ and there are no special orbits, the root systems $\Sigma_{t \sigma}$ and $\Phi_{t \sigma}$ are dual to each other.

Proof. This is an immediate consequence of the definitions: when $n_{\sigma}>1$ then $\mathbf{G}$ is of type $A D E$ thus $\Sigma \simeq \Sigma^{\vee}$, and via this isomorphism the definitions of $\Phi_{t \sigma}$ and $\Sigma_{t \sigma}^{\vee}$ match, as well as those of $\Phi_{t \sigma}^{\vee}$ and $\Sigma_{t \sigma}$. 


\section{ISOLATED AND QUASI-ISOLATED ELEMENTS}

Definition 3.1. We say that the quasi-semisimple element $t \sigma$ is isolated if $\left(\mathbf{G}^{t \sigma}\right)^{0}$ is not contained in a $\sigma$-stable Levi subgroup of a proper $\sigma$-stable parabolic subgroup of $\mathbf{G}$.

Proposition 3.2. The following are equivalent:

(1) $t \sigma$ is isolated.

(2) Every central torus in $\left(\mathbf{G}^{t \sigma}\right)^{0}$ is central in $\mathbf{G}$.

(3) $Z^{0}\left(\left(\mathbf{G}^{t \sigma}\right)^{0}\right)=Z^{0}\left(\left(\mathbf{G}^{\sigma}\right)^{0}\right)$.

(4) $\Sigma_{t \sigma}$ and $\Sigma_{\sigma}$ span the same subspace of $X\left(\left(\mathbf{T}^{\sigma}\right)^{0}\right) \otimes \mathbb{Q}$.

(4') $\Phi_{t \sigma}$ and $\Phi_{\sigma}$ span the same subspace of $X\left(\left(\mathbf{T}^{\sigma}\right)^{0}\right) \otimes \mathbb{Q}$.

(5) $W^{0}(t \sigma)$ is not in a proper parabolic subgroup of $W^{\sigma}$.

Proof. Let us prove $(1) \Leftrightarrow(2)$. If (1) fails then $\left(\mathbf{G}^{t \sigma}\right)^{0}$ is contained in a proper $\sigma$ stable Levi subgroup $\mathbf{L}$ of a $\sigma$-stable parabolic subgroup of $\mathbf{G}$. In particular, by DM94, 1.25] $\left(\mathbf{L}^{\sigma}\right)^{0}$ is a proper Levi subgroup of $\left(\mathbf{G}^{\sigma}\right)^{0}$ and $Z^{0}\left(\left(\mathbf{L}^{\sigma}\right)^{0}\right)$ is central in $\mathbf{L}$ but not in $\mathbf{G}$ by [DM94, 1.23]. By [DM94, 1.8(iii)] $\left(\mathbf{T}^{\sigma}\right)^{0}=\left(\mathbf{T}^{t \sigma}\right)^{0}$ is a maximal torus of $\left(\mathbf{G}^{t \sigma}\right)^{0}$, thus it is a maximal torus of $\left(\mathbf{L}^{\sigma}\right)^{0}$, and $Z^{0}\left(\left(\mathbf{L}^{\sigma}\right)^{0}\right)$ is in this torus thus in $\left(\mathbf{G}^{t \sigma}\right)^{0}$; and $Z^{0}\left(\left(\mathbf{L}^{\sigma}\right)^{0}\right)$ is central in $\left(\mathbf{G}^{t \sigma}\right)^{0}$ since it is central in $\mathbf{L}$. Thus (2) fails.

If (2) fails, then there is a torus $\mathbf{S}$ central in $\left(\mathbf{G}^{t \sigma}\right)^{0}$ not central in $\mathbf{G}$; the torus $\mathbf{S}$ is in all maximal tori of $\left(\mathbf{G}^{t \sigma}\right)^{0}$, in particular in $\left(\mathbf{T}^{\sigma}\right)^{0}$ thus $\mathbf{S} \subset\left(\mathbf{G}^{\sigma}\right)^{0}$; thus by [DM94, 1.25(ii)], $C_{\mathbf{G}}(\mathbf{S})$ is a Levi subgroup of a $\sigma$-stable proper parabolic subgroup of $\mathbf{G}$ containing $\left(\mathbf{G}^{t \sigma}\right)^{0}$.

Let us prove $(2) \Leftrightarrow(3)$. We first observe that one has always $Z^{0}\left(\left(\mathbf{G}^{t \sigma}\right)^{0}\right) \supset$ $Z^{0}\left(\left(\mathbf{G}^{\sigma}\right)^{0}\right)$ since by Proposition 1.28 we have $Z^{0}\left(\left(\mathbf{G}^{\sigma}\right)^{0}\right)=\left((Z \mathbf{G})^{\sigma}\right)^{0}$, and since $Z \mathbf{G} \subset \mathbf{T}$, we have $\left.\left((Z \mathbf{G})^{\sigma}\right)^{0}\right)=\left((Z \mathbf{G})^{t \sigma}\right)^{0}$. Now if $(2)$ holds every element of $Z^{0}\left(\left(\mathbf{G}^{t \sigma}\right)^{0}\right)$ is central in $\mathbf{G}$ and contained in $\left(\mathbf{T}^{\sigma}\right)^{0}$, thus central in $\left(\mathbf{G}^{\sigma}\right)^{0}$, whence equality. Conversely, if there is equality any torus central in $\left(\mathbf{G}^{t \sigma}\right)^{0}$ is central in $\left(\mathbf{G}^{\sigma}\right)^{0}$, thus in $\mathbf{G}$ since $Z^{0}\left(\left(\mathbf{G}^{\sigma}\right)^{0}\right) \subset Z^{0}(\mathbf{G})$ by [DM94, 1.23] applied with $\mathbf{L}=\mathbf{G}$.

Let us prove $(3) \Leftrightarrow(4)$. It is a general fact in a reductive group $\mathbf{G}$ that if $\Sigma^{\perp}$ is the orthogonal of $\Sigma$ in $Y \otimes \mathbb{Q}$ then $Y\left(Z^{0} \mathbf{G}\right)=Y \cap \Sigma^{\perp}$ and generates $\Sigma^{\perp}$. Applying this to $\left(\mathbf{G}^{t \sigma}\right)^{0},(3)$ can be rewritten $\Sigma_{t \sigma}^{\perp}=\Sigma_{\sigma}^{\perp}$ where $\perp$ is taken in $Y\left(\left(\mathbf{T}^{\sigma}\right)^{0}\right) \otimes \mathbb{Q}$. We get (4) by taking the orthogonal of this equality.

(4) is equivalent to $\left(4^{\prime}\right)$ since $\Phi_{t \sigma}$ and $\Sigma_{t \sigma}$ span the same $\mathbb{Q}$-vector space, as do $\Phi_{\sigma}$ and $\Sigma_{\sigma}$.

We finally prove $(4) \Leftrightarrow(5)$. We have $W^{\sigma}=W^{0}(\sigma)$ by Proposition 1.20 , The equivalence of (4) and (5) thus follows from the fact that $W^{0}(t \sigma)$ and $W^{0}(\sigma)$ are the Weyl groups of the root systems $\Sigma_{t \sigma}$ and $\Sigma_{\sigma}$ respectively.

Corollary 3.3. If $\mathbf{G}$ is semisimple and to isolated, then $\mathbf{G}^{t \sigma}$ is semisimple.

Proof. This is a straightforward consequence of Proposition $3.2(2)$.

Remark 3.4. Proposition 3.2 (3) fails if one does not take the connected components, that is we do not have in general $Z\left(\mathbf{G}^{\sigma}\right) \subset Z\left(\mathbf{G}^{t \sigma}\right)$, as the following example shows: take $\mathbf{G}=\mathrm{GL}_{3}$ with $\sigma$ of symplectic type and $t$ such that $t \sigma$ is of orthogonal type; then $\mathbf{G}^{\sigma} \simeq \mathrm{Sp}_{2} \times\{ \pm 1\}$ and $\mathbf{G}^{t \sigma} \simeq \mathrm{O}_{3}$; we have $Z\left(\mathbf{G}^{\sigma}\right) \simeq(\mathbb{Z} / 2 \mathbb{Z})^{2}$ and $Z\left(\mathbf{G}^{t \sigma}\right) \simeq \mathbb{Z} / 2 \mathbb{Z}$. 
To study isolated classes we can assume $\mathbf{G}$ to be semisimple, thanks to the following proposition.

Proposition 3.5. We have $\left(\mathbf{T}^{\sigma}\right)^{0}=\left((Z \mathbf{G})^{\sigma}\right)^{0}\left((\mathbf{T} \cap D \mathbf{G})^{\sigma}\right)^{0}$ and if we write $t \in$ $\left(\mathbf{T}^{\sigma}\right)^{0}$ as $t=z t_{1}$ with $z \in\left((Z \mathbf{G})^{\sigma}\right)^{0}$ and $t_{1} \in\left((\mathbf{T} \cap D \mathbf{G})^{\sigma}\right)^{0}$ then $\mathbf{G}^{t \sigma}=\mathbf{G}^{t_{1} \sigma}$ and t $\sigma$ is isolated in $\mathbf{G} \cdot \sigma$ if and only if $t_{1} \sigma$ is isolated in $D \mathbf{G} \cdot \sigma$.

Proof. We claim that

$$
\left(\mathbf{G}^{t \sigma}\right)^{0}=\left((Z \mathbf{G})^{\sigma}\right)^{0}\left((D \mathbf{G})^{t \sigma}\right)^{0} .
$$

Indeed, by DM94, 1.31] the index of $(Z \mathbf{G})^{\sigma}(D \mathbf{G})^{t \sigma}$ in $\mathbf{G}^{t \sigma}$ is finite so that the identity components coincide. Since $\left((Z \mathbf{G})^{\sigma}\right)^{0} \subset\left(\mathbf{T}^{\sigma}\right)^{0}$, we deduce that any $t \in$ $\left(\mathbf{T}^{\sigma}\right)^{0}$ can be written $t=z t_{1}$ with $z \in\left((Z \mathbf{G})^{\sigma}\right)^{0}$ and $t_{1} \in\left((\mathbf{T} \cap D \mathbf{G})^{\sigma}\right)^{0}$. We then have $\mathbf{G}^{t \sigma}=\mathbf{G}^{t_{1} \sigma}$ since $z$ commutes with $\mathbf{G}$ and with $\sigma$. Hence $t \sigma$ is isolated in $\mathbf{G} \cdot \sigma$ if and only if $t_{1} \sigma$ is isolated in $\mathbf{G} \cdot \sigma$ which is equivalent to $t_{1} \sigma$ being isolated in $D \mathbf{G} \cdot \sigma$ since by Equation 3.6 applied with $t_{1}$ instead of $t$ the Weyl group $W^{0}\left(t_{1} \sigma\right)$ is the same in $\mathbf{G}$ and in $D \mathbf{G}$.

Proposition 3.7. If the image of $\sigma \sigma$ in $\mathbf{T} /[\mathbf{T}, \sigma]$ is parameterized by $\lambda \in \mathcal{C}$, and $S_{t \sigma}$ is the set of reflections with respect to the walls of $\mathcal{C}$ containing $\lambda$, then $\left(W^{0}(t \sigma), S_{t \sigma}\right)$ is a Coxeter system. The subset $\Delta_{t \sigma}$ of $\tilde{\Delta}_{\sigma}$ corresponding to the reflections in $S_{t \sigma}$ is a basis of $\Phi_{t \sigma}$.

Proof. If $\lambda \in \mathcal{C}$, by Proposition 2.7 and Bbk. Chap. V $\S 3$ no 3, (vii) of Proposition $1]$, a set of generators of $W^{0}(t \sigma)$ is given by the reflections with respect to the walls of $\mathcal{C}$ containing $\lambda$. The set $S_{t \sigma}$ of these reflections is a subset of $\tilde{S}_{\sigma}$, thus $\left(W^{0}(t \sigma), S_{t \sigma}\right)$ is a parabolic subsystem of $\left(\tilde{S}_{\sigma}, W_{a}\right)$.

To see that $\Delta_{t \sigma}$ is a basis of $\Phi_{t \sigma}$, we note that for any subset of $\tilde{S}_{\sigma}$ a basis of the corresponding parabolic subsystem is given by the corresponding subset of $\tilde{\Delta}_{\sigma}$. This is a consequence of the following lemma

Lemma 3.8. Let $\Delta$ be a basis of a root system $\Phi$ and let $\alpha_{0}$ be the highest root of $\Phi$; then for any $\alpha \in \Delta$ the set $\Delta_{\alpha}:=\left(\Delta \cup\left\{-\alpha_{0}\right\}\right)-\{\alpha\}$ is a basis of the root subsystem it generates.

Proof. Let $\Delta:=\left\{\alpha_{1}, \ldots, \alpha_{r}\right\}$ with $\alpha=\alpha_{1}$ and let $\alpha_{0}=\sum_{i} n_{i} \alpha_{i}$. Let $\beta \in \Phi^{+}$be of the form $\sum_{\alpha_{i} \in \Delta_{\alpha}} m_{i} \alpha_{i}$ with $m_{i} \in \mathbb{Z}_{\geq 0}$. We have $\beta=-\frac{m_{1}}{n_{1}}\left(-\alpha_{0}\right)+\sum_{i \geq 2}\left(m_{i}-\right.$ $\left.n_{i} \frac{m_{1}}{n_{1}}\right) \alpha_{i}$. Since $\alpha_{0}$ is the highest root we have $0 \leq \frac{m_{1}}{n_{1}} \leq 1$. As $\frac{m_{1}}{n_{1}}$ is an integer, it is equal to 0 or 1 . If $m_{1}=n_{1}$ we have $\beta=-\left(-\alpha_{0}\right)+\sum_{i \geq 2}\left(m_{i}-n_{i}\right) \alpha_{i}$ and all the coefficients are non-positive. If $m_{1}=0$ all coefficients are non negative.

Proposition 3.9. Assume $\mathbf{G}^{\sigma}$ semisimple, so that every conjugacy class of quasisemisimple elements in $\mathbf{G} \cdot \sigma$ has a representative parameterized by $\lambda \in \mathcal{C}$.

If $t \sigma$ is such a representative, then $t \sigma$ is isolated if and only if $\left|\tilde{S}_{\sigma, i}-S_{t \sigma}\right|=1$ for each irreducible component $S_{\sigma, i}$ of $S_{\sigma}$.

Conversely, if $\Omega$ is a subset of $\left(\tilde{S}_{\sigma}\right)_{p^{\prime}}:=\left\{s \in \tilde{S}_{\sigma} \mid \frac{1}{n_{s}} \varpi_{s}^{\vee} \in Y_{\sigma} \otimes \mathbb{Q}_{p^{\prime}}\right\}$ such that $\left|\Omega \cap \tilde{S}_{\sigma, i}\right|=1$ for each $i$, let $\left\{s_{i}\right\}=\tilde{S}_{\sigma, i} \cap \Omega$, and let $\varpi_{s_{i}}^{\vee}$ be the corresponding fundamental coweight (where by convention $\varpi_{s_{0, i}}^{\vee}=0$ ). Then $\sum_{i} \frac{1}{n_{s_{i}}} \varpi_{s_{i}}^{\vee}$ is the unique element of $\mathcal{C}$ such that for a corresponding to we have $S_{t \sigma}=\cup_{i}\left(\tilde{S}_{\sigma, i}-\left\{\alpha_{s_{i}}\right\}\right)$. 
It follows that the $\mathbf{G}$-conjugacy classes of isolated elements of finite order of $\mathbf{G} \cdot \sigma$ are parameterized by the $\widetilde{W} / W_{a}$-orbits of subsets $\Omega$ of $\left(\tilde{S}_{\sigma}\right)_{p^{\prime}}$ such that $\left|\Omega \cap \tilde{S}_{\sigma, i}\right|=1$ for each $i$; the action of $\widetilde{W} / W_{a}$ comes from the embedding $\widetilde{W} \subset W_{a}^{\prime}-$ which exists since $\mathbf{G}^{\sigma}$ is semisimple - and from the identification of $W_{a}^{\prime} / W_{a}$ to a group of automorphisms of $\mathcal{C}$, see Proposition 2.5(5).

Proof. By Proposition 3.7 $\left(W^{0}(t \sigma), S_{t \sigma}\right)$ is a Coxeter system. The class of $t \sigma$ is isolated if and only if $S_{t \sigma}$ has same rank as $W$, that is if and only if, setting $\Omega:=\tilde{S}-S_{t \sigma}$, we have $\left|\Omega \cap \tilde{S}_{\sigma, i}\right|=1$ for each irreducible component $S_{\sigma, i}$ of $S_{\sigma}$.

Conversely such a set $\Omega \subset \tilde{S}_{\sigma}$ defines a unique point $\lambda \in \mathcal{C}$ namely the intersection of the walls of $\mathcal{C}$ corresponding to $\tilde{S}-\Omega$ : indeed, since in each irreducible component we consider all the walls but one, such a $\lambda$ is the product of extremal points of the simplices $\mathcal{C}_{i}$, hence, by Proposition 2.5 (2), is equal to $\sum_{i} \frac{1}{n_{s_{i}}} \varpi_{s_{i}}^{\vee}$.

We will need the following lemma in the proof of the next theorem.

Lemma 3.10. If $\mathbf{H}$ is a connected reductive group and $\mathbf{K}$ is a closed subgroup of $Z \mathbf{H}$, then $Z(\mathbf{H} / \mathbf{K})=(Z \mathbf{H}) / \mathbf{K}$.

Proof. Let $\mathbf{T}$ be a maximal torus of $\mathbf{H}$. The roots of $\mathbf{H}$ are the inflations to $\mathbf{T}$ of the roots of $\mathbf{H} / \mathbf{K}$ with respect to $\mathbf{T} / \mathbf{K}$ since the root subgroups of $\mathbf{H}$ are mapped isomorphically to the root subgroups of $\mathbf{H} / \mathbf{K}$. The center of $\mathbf{H} / \mathbf{K}$ is the intersection of the kernels of the roots, and $\mathbf{K}$ lies in that intersection, whence the result.

In what follows, we write $e(G)$ for the exponent of a group $G$ and $e(x)$ for the order of $x \in G$. For an algebraic group $\mathbf{G}$, we set $\mathrm{AZ}(\mathbf{G}):=Z(\mathbf{G}) / Z^{0}(\mathbf{G})$.

Theorem 3.11. As in Context 1.23, let $\sigma$ be quasi-central inducing an automorphism of order $n_{\sigma}$ and let $t \in\left(\mathbf{T}^{\sigma}\right)^{0}$ be of finite order $e(t)$; if $Z \mathbf{G}$ is connected then $e\left(\mathrm{AZ}\left(\left(\mathbf{G}^{t \sigma}\right)^{0}\right)\right)$ divides $n_{\sigma} e(t)$.

Proof. We prove this by a series of reductions.

- The first step is to reduce to the case where $t \sigma$ is isolated. Otherwise $\left(\mathbf{G}^{t \sigma}\right)^{0}$ is in a proper Levi subgroup of $\mathbf{G}$ and we may replace $\mathbf{G}$ with this proper Levi subgroup without changing $\left(\mathbf{G}^{t \sigma}\right)^{0}$ or $t \sigma$.

- The next step is to assume that $Z \mathbf{G}=[Z \mathbf{G}, \sigma]$. Indeed we may quotient $\mathbf{G}$ by $\left((Z \mathbf{G})^{\sigma}\right)^{0} \subset Z^{0}\left(\left(\mathbf{G}^{t \sigma}\right)^{0}\right)$ which does not change $\mathrm{AZ}\left(\left(\mathbf{G}^{t \sigma}\right)^{0}\right)$ nor $n_{\sigma}$ and can only replace $e(t)$ with a divisor. The center of $\mathbf{G} /\left((Z \mathbf{G})^{\sigma}\right)^{0}$ is $Z^{\prime}:=Z \mathbf{G} /\left((Z \mathbf{G})^{\sigma}\right)^{0}$ by Lemma 3.10 and satisfies $Z^{\prime}=\left[Z^{\prime}, \sigma\right]$ since $Z \mathbf{G}=\left((Z \mathbf{G})^{\sigma}\right)^{0}[Z \mathbf{G}, \sigma]$.

- The next step is to reduce to the case $\mathbf{G}$ adjoint. If $\mathbf{G}_{\mathrm{ad}}=\mathbf{G} / Z \mathbf{G}$ then we have an exact sequence $1 \rightarrow(Z \mathbf{G})^{\sigma} \rightarrow \mathbf{G}^{t \sigma} \rightarrow \mathbf{G}_{\text {ad }}^{t \sigma} \rightarrow 1$; indeed the only nontrivial point is the surjectivity; if $\bar{g} \in \mathbf{G}_{\text {ad }}^{t \sigma}$ then it is the image of $g \in \mathbf{G}$ such that $g^{-1 t \sigma} g \in Z \mathbf{G}$, and by the equality $Z \mathbf{G}=[Z \mathbf{G}, \sigma]$ we may write $g^{-1 t \sigma} g=z^{-1 t \sigma} z$ with $z \in Z \mathbf{G}$, thus $g z^{-1} \in \mathbf{G}^{t \sigma}$ has same image in $\mathbf{G}_{\mathrm{ad}}$ as $g$.

Taking the identity components, the above exact sequence gives the exact sequence $1 \rightarrow Z \mathbf{G} \cap\left(\mathbf{G}^{t \sigma}\right)^{0} \rightarrow\left(\mathbf{G}^{t \sigma}\right)^{0} \rightarrow\left(\mathbf{G}_{\mathrm{ad}}^{t \sigma}\right)^{0} \rightarrow 1$. Taking centers, we get by Lemma 3.10 the exact sequence $1 \rightarrow Z \mathbf{G} \cap\left(\mathbf{G}^{t \sigma}\right)^{0} \rightarrow Z\left(\left(\mathbf{G}^{t \sigma}\right)^{0}\right) \rightarrow Z\left(\left(\mathbf{G}_{\text {ad }}^{t \sigma}\right)^{0}\right) \rightarrow 1$.

Since we have $\left(Z \mathbf{G} \cap\left(\mathbf{G}^{t \sigma}\right)^{0}\right)^{0}=\left((Z \mathbf{G})^{t \sigma}\right)^{0}=\left((Z \mathbf{G})^{\sigma}\right)^{0}$, the exponent of $(Z \mathbf{G} \cap$ $\left.\left(\mathbf{G}^{t \sigma}\right)^{0}\right) /\left(Z \mathbf{G} \cap\left(\mathbf{G}^{t \sigma}\right)^{0}\right)^{0}$ divides that of $(Z \mathbf{G})^{\sigma} /\left((Z \mathbf{G})^{\sigma}\right)^{0}$, and the exponent of this last group divides $n_{\sigma}$ by Lemma 1.2 it follows that $e\left(\mathrm{AZ}\left(\mathbf{G}^{t \sigma}\right)^{0}\right)$ divides the $1 \mathrm{~cm}$ of $n_{\sigma}$ and $e\left(\mathrm{AZ}\left(\left(\mathbf{G}_{\mathrm{ad}}^{t \sigma}\right)^{0}\right)\right)$. It is thus sufficient to prove the theorem for $\mathbf{G}_{\mathrm{ad}}$. 
- The next step is to reduce to the case $\mathbf{G}$ simple. Indeed, since $\mathbf{G}$ is adjoint, it is uniquely a direct product of simple groups, permuted by $\sigma$. If we consider a subset of $i$ components cyclically permuted by $\sigma$, the group of fixed points under $t \sigma$ is isomorphic to the group of fixed points under $(t \sigma)^{i}$ in one component. Since $n_{\sigma} e(t)$ (resp. $\left.e\left(\mathrm{AZ}\left(\mathbf{G}^{t \sigma}\right)^{0}\right)\right)$ is the lcm of the analogous numbers for each component, it is enough to show the theorem for a simple group.

- For a simple group we use the description of isolated elements in Proposition 3.9. thus $t \sigma$ has same centralizer as an element of finite order whose image $\bar{t}$ in $\mathbf{T} /[\mathbf{T}, \sigma]$ is parameterized by $\lambda=\frac{1}{n_{s}} \varpi_{s}^{\vee} \in Y_{\sigma} \otimes \mathbf{Q}_{p^{\prime}}$ where $\Omega=\{s\}$. If $z$ is central in $\left(\mathbf{G}^{t \sigma}\right)^{0}$ then $\left(\mathbf{G}^{z t \sigma}\right)^{0} \supseteq\left(\mathbf{G}^{t \sigma}\right)^{0}$, thus $W^{0}(t \sigma) \subseteq W^{0}(z t \sigma)$. By Proposition 2.7 we deduce $\Phi_{t \sigma} \subseteq \Phi_{z t \sigma}$. Since $\Phi_{t \sigma}$ (resp. $\Phi_{z t \sigma}$ ) consists of the roots of $\Phi_{\sigma}$ such that $\alpha(\lambda) \in \mathbb{Z}$ (resp. $\alpha(\mu) \in \mathbb{Z}$ if $\mu$ parameterizes the image of $t z$ in $\mathbf{T} /[\mathbf{T}, \sigma]$ ), we have $\alpha(\mu) \in \mathbb{Z}$ if $\alpha(\lambda) \in \mathbb{Z}$. It follows that $\mu=\frac{a}{n_{s}} \varpi_{s}^{\vee}$ modulo $Y_{\sigma}$ for some $a \in \mathbb{Z}$, thus the image $\overline{t z}$ of $t z$ in $\mathbf{T} /[\mathbf{T}, \sigma]$ is a power of $\bar{t}$. Thus $e(\bar{z})$ divides $e(\bar{t})$.

Since the kernel of the map $\left(\mathbf{T}^{\sigma}\right)^{0} \rightarrow \mathbf{T} /[\mathbf{T}, \sigma]$ has exponent dividing $n_{\sigma}$ by Lemma 1.2, it follows that $e(z)$ divides $n_{\sigma} e(t)$.

\section{Quasi-isolated elements.}

Definition 3.12. We say that the quasi-semisimple element $t \sigma$ is quasi-isolated if $\mathbf{G}^{t \sigma}$ is not contained in a $\sigma$-stable Levi subgroup of a proper $\sigma$-stable parabolic subgroup of $\mathbf{G}$.

In particular an isolated element is quasi-isolated.

Proposition 3.13. The following are equivalent:

(1) $t \sigma$ is quasi-isolated.

(2) $W(t \sigma)$ is not in a proper parabolic subgroup of $W^{\sigma}$.

Proof. The parabolic subgroups of the Weyl group of a reductive group are in oneto-one correspondence with the Levi subgroups containing a given maximal torus. Applying this in $\left(\mathbf{G}^{\sigma}\right)^{0}$ we deduce from [DM94, 1.25(ii)] that parabolic subgroups of $W^{\sigma}$ are in one-to-one correspondence with the $\sigma$-stable Levi subgroups containing $\mathbf{T}$ of $\sigma$-stable parabolic subgroups of $\mathbf{G}$.

We prove that (1) implies (2). If (2) fails, by the above $W(t \sigma)$ has representatives in a $\sigma$-stable Levi subgroup $\mathbf{L}$ of a proper $\sigma$-stable parabolic subgroup. By the proofs of Proposition 1.12 and Proposition 1.14, $\mathbf{G}^{t \sigma}$ is generated by $\left(\mathbf{G}^{t \sigma}\right)^{0}$ and representatives of $W(t \sigma)$. By the equivalence of (1) and (5) in Proposition 3.2 the group $\left(\mathbf{G}^{t \sigma}\right)^{0}$ is contained in the Levi subgroup $\mathbf{L}$, thus the whole of $\mathbf{G}^{t \sigma}$ is contained in a proper Levi subgroup, that is $t \sigma$ is not quasi-isolated.

We prove that (2) implies (1). If (1) fails then $\mathbf{G}^{t \sigma}$ is contained in a $\sigma$-stable Levi subgroup $\mathbf{L}$ of a proper $\sigma$-stable parabolic subgroup, hence representatives of all elements of $W(t \sigma)$ can be taken in $\mathbf{L}$, thus $W(t \sigma)$ is contained in the Weyl group of $\mathbf{L}$ and (2) fails.

Proposition 3.14. If the class of $t \sigma$ is parameterized by $\lambda$ (see Theorem 1.17) then $W(t \sigma)=\left\{w \in W^{\sigma} \mid w(\lambda)-\lambda \in Y_{\sigma}\right\}$.

Proof. The class of $t \sigma$ being parameterized by $\lambda \in Y_{\sigma} \otimes \mathbb{Q}$ means that the image $\bar{t}$ of $t$ in $\mathbf{T} /[\mathbf{T}, \sigma]$ is identified with the image $\bar{\lambda}$ of $\lambda$ in $Y_{\sigma} \otimes \mathbb{Q} / \mathbb{Z}$ (see the proof of Theorem 1.17); hence $w \in W^{\sigma}$ fixes $\bar{t}$ if and only if it fixes $\bar{\lambda}$, whence the assertion. 
Remark 3.15. The following example shows that an element quasi-isolated in $\mathbf{G} \cdot \sigma$ may not be quasi-isolated in $D(\mathbf{G}) \cdot \sigma$, which forbids taking the derived group as a method to reduce the study of quasi-isolated elements to the case of semisimple groups. Let $\mathbf{G}=\mathrm{GL}_{2}$, thus $D(\mathbf{G})=\mathrm{SL}_{2}$. Let us take $\sigma$ quasi-central defined on G by $\sigma(x)=w^{t}\left(x^{-1}\right) w^{-1}$ where $w=\left(\begin{array}{cc}0 & 1 \\ -1 & 0\end{array}\right)$. We have $\left(\mathbf{G}^{\sigma}\right)^{0}=D(\mathbf{G})$. Let $t=\left(\begin{array}{cc}i & 0 \\ 0 & -i\end{array}\right)$. Then $W^{0}(t \sigma)=1$, and $W(t \sigma)=1$ in $D(\mathbf{G})$ but $W(t \sigma)=W^{\sigma}=W$ in $\mathbf{G}$. The centralizer of $t \sigma$ in $D(\mathbf{G})=\mathrm{SL}_{2}$ is the maximal torus $\mathbf{T}^{\prime}=\operatorname{diag}\left(a, a^{-1}\right)$, but $\mathbf{G}^{t \sigma}$ is generated by $\mathbf{T}^{\prime}$ and $\left(\begin{array}{ll}0 & 1 \\ 1 & 0\end{array}\right)$. Thus $t \sigma$ is quasi-isolated in $\mathbf{G} \cdot \sigma$ but not in $D(\mathbf{G}) \cdot \sigma$. The "explanation" is that $Y_{\sigma}$ is generated by $\alpha^{\vee} / 2$ in the $\mathrm{GL}_{2}$ case, and by $\alpha^{\vee}$ in the $\mathrm{SL}_{2}$ case, so applying Proposition 3.14 yields different results equivalently $[\mathbf{T}, \sigma]$ is trivial in $\mathrm{SL}_{2}$ but is equal to the center in $\mathrm{GL}_{2}$, so using the definition of $W(t \sigma)$ one gets different results.

Definition 3.16. We define $\mathcal{A}_{t \sigma}:=\left\{w \in W(t \sigma) \mid w\left(\Delta_{t \sigma}\right)=\Delta_{t \sigma}\right\}$.

Proposition 3.17. $W(t \sigma)=W^{0}(t \sigma) \rtimes \mathcal{A}_{t \sigma}$.

Proof. By Proposition 3.7, $\Delta_{t \sigma}$ is a basis of the root system of $W^{0}(t \sigma)$. Since two such bases are conjugate under $W^{0}(t \sigma)$, and $W^{0}(t \sigma)$ is the (normal) subgroup generated by the reflections of $W(t \sigma)$ (see Proposition 2.7 and Proposition 3.14) we get the semi-direct decomposition.

Proposition 3.18. The group $\mathcal{A}_{t \sigma}$ embeds in $\mathcal{A}_{R(\sigma)}:=\left(Y_{\sigma} \cap \mathbb{Q} \Phi_{\sigma}^{\vee}\right) / Q\left(\Phi_{\sigma}^{\vee}\right)$.

Proof. Consider the map $W(t \sigma) \rightarrow Y_{\sigma} / Q\left(\Phi_{\sigma}^{\vee}\right): w \mapsto w(\lambda)-\lambda\left(\bmod Q\left(\Phi_{\sigma}^{\vee}\right)\right)$ where $t \sigma$ is parameterized by $\lambda$, see Theorem 1.17. Its image lies in $\left(Y_{\sigma} \cap \mathbb{Q} \Phi_{\sigma}^{\vee}\right) / Q\left(\Phi_{\sigma}^{\vee}\right)$ since $w(\lambda)-\lambda$ is a $\mathbb{Q}$-linear combination of coroots. Since $W^{\sigma}$ is generated by the reflections with respect to $\Phi_{\sigma}$ by Proposition 2.3. using the formula defining a reflection we see that $W^{\sigma}$ acts trivially on $Y_{\sigma} / Q\left(\Phi_{\sigma}^{\vee}\right)$, hence the above map is a group morphism. Its kernel is $\left\{w \in W^{\sigma} \mid w(\lambda)-\lambda \in Q\left(\Phi_{\sigma}^{\vee}\right)\right\}$ which is a reflection group by [Bbk, Ch. VI ex. 1 of $\S 2$ ], hence is generated by the reflections satisfying the same condition, thus is equal to $W^{0}(t \sigma)$ by Proposition 2.7

Note that by Proposition $2.5(5), \mathcal{A}_{R(\sigma)}$ can be identified to a subgroup of $\operatorname{Aut}_{W^{\sigma}}\left(\tilde{\Delta}_{\sigma}\right)$.

Remark 3.19. In view of Proposition 3.17 and Proposition 3.18 if $\mathcal{A}_{R(\sigma)}=1$ (which can be stated as "the derived root datum of $R(\sigma)$ is simply connected") then $W(t \sigma)=W^{0}(t \sigma)$ and every quasi-isolated element is isolated.

Proposition 3.20. Let $t \sigma$ be an element parameterized by $\lambda \in \mathcal{C}$, then $t \sigma$ is quasiisolated if and only if $\mathcal{A}_{t \sigma}$ acts transitively on $\tilde{S}_{\sigma, i}-S_{t \sigma}$ for every $i$.

For such an element, $\mathcal{A}_{t \sigma}$ is the stabilizer of $\Delta_{t \sigma}$ in $\mathcal{A}_{R(\sigma)}$.

Proof. We show that we can apply B05. Corollary 4.3(b)]. B05 considers the Weyl group $W$ of a root system $\Phi$ acting on a lattice $Y(\mathbf{T})$ and on the space $V=Y(\mathbf{T}) \otimes \mathbb{Q}$, the affine group $W_{\text {aff }}=W \rtimes \mathbb{Z} \Phi$, the fundamental domain $\mathcal{C}$ of $W_{\text {aff }}$ on $V$ and an element $\lambda \in \mathcal{C}$. We can specialize these objects to correspond to our setting as follow:

$$
\begin{array}{ccccccc}
W & \Phi & Y(\mathbf{T}) & V & W_{\text {aff }} & \mathcal{C} & \lambda \\
W^{\sigma} & \Phi_{\sigma} & Y_{\sigma} \cap \mathbb{Q} \Phi_{\sigma}^{\vee} & \mathbb{Q} \Phi_{\sigma}^{\vee} & W_{a} & \mathcal{C} & \lambda
\end{array}
$$


Then, comparing our Proposition 3.14, Proposition 2.7 to the definitions B05, below Lemma 3.1], we can extend this dictionary as follows

$$
\begin{array}{ccc}
W_{\mathbf{G}}(\lambda) & W^{0}(\lambda) & \Phi(\lambda) \\
W(t \sigma) & W^{0}(t \sigma) & \Phi_{t \sigma}
\end{array}
$$

Then comparing our Proposition 3.7 to [B05, proof of 3.5], our Proposition 3.18 to [B05, 3.C], and our Proposition [3.7 to [B05, Proposition 3.14] we have the correspondences

$$
\begin{array}{ccc}
\tilde{\Delta} & \mathcal{A}_{\mathbf{G}} & I_{\lambda} \\
\tilde{\Delta}_{\sigma} & \mathcal{A}_{R(\sigma)} & \Delta_{t \sigma}
\end{array}
$$

Now by [B05, below Lemma 3.1] joint with the choice made in [B05, last line before section 4] $A_{\mathrm{G}}(\lambda)$ of loc. cit. is our $\mathcal{A}_{t \sigma}$.

We could have equivalently given the first sentence of Proposition 3.20 in terms of the action of $\mathcal{A}_{t \sigma}$ on $\Delta_{t \sigma}$, the condition for a quasi-semisimple element to be quasiisolated given in Proposition 3.13 becoming that given below Lemma 3.2 in B05, we can use [B05. Corollary 4.3 (b)] which gives the first sentence of Proposition 3.20 .

To prove the last sentence of Proposition 3.20, following [B05] we introduce affine coordinates $\left\{\lambda_{\alpha}\right\}_{\alpha \in \tilde{\Delta}}$ for $\lambda$ as in [B05, below (3.9)]. Then by [B05, 3.14(b)] we have

$$
\mathcal{A}_{t \sigma}=\left\{a \in \mathcal{A}_{R(\sigma)} \mid \forall \alpha \in \tilde{\Delta}, \lambda_{a(\alpha)}=\lambda_{\alpha}\right\} .
$$

Since by the first sentence of Proposition $3.20 \mathcal{A}_{t \sigma}$ acts transitively on $\tilde{\Delta}-\Delta_{t \sigma}$, it follows that $\lambda_{\alpha}$ is constant (and non-zero by [B05, 3.14(a)]) on this set. Since $\lambda_{\alpha}$ is constant equal to 0 on $\Delta_{t \sigma}$ (by [B05, 3.14(a)]), it is necessary and sufficient to stabilize $\Delta_{t \sigma}$ in order to satisfy the condition on the right-hand side of $(*)$, whence the second sentence of Proposition 3.20 .

Proposition 3.21. Assume $\mathbf{G}^{\sigma}$ semisimple, so that every conjugacy class of quasisemisimple elements in $\mathbf{G} \cdot \sigma$ has a representative parameterized by $\lambda \in \mathcal{C}$. Then:

If $\Omega$ is a subset of $\left(\tilde{S}_{\sigma}\right)_{p^{\prime}}:=\left\{s \in \tilde{S}_{\sigma} \mid \frac{1}{n_{s}} \varpi_{s}^{\vee} \in \mathbb{Z}_{(p)} \Phi_{\sigma}^{\vee}\right\}$ such that the stabilizer in $\mathcal{A}_{R(\sigma)}$ of $\Omega \cap \tilde{S}_{\sigma, i}$ acts transitively on it for each $i$, the element $\sum_{i} \frac{\sum_{s \in \Omega \cap \tilde{S}_{i}} \varpi_{s}^{\vee}}{n_{i}(\Omega)\left|\Omega \cap \Delta_{\sigma, i}\right|}$, where $n_{i}$ is the common value of $n_{s}$ for every $s \in \Omega \cap \tilde{S}_{i}$, is the unique point of $\mathcal{C}$ such that for a corresponding to we have $S_{t \sigma}=\cup_{i}\left(\tilde{S}_{\sigma, i}-\Omega\right)$ and $\mathcal{A}_{t \sigma}$ acts transitively on $\tilde{S}_{\sigma, i}-S_{t \sigma}$.

The $\mathbf{G}$-conjugacy classes of quasi-isolated elements of finite order of $\mathbf{G} \cdot \sigma$ are parameterized by the $\widetilde{W} / W_{a}$-orbits of subsets $\Omega$ of $\left(\tilde{S}_{\sigma}\right)_{p^{\prime}}$ such that the stabilizer in $\mathcal{A}_{R(\sigma)}$ of $\Omega \cap \tilde{S}_{\sigma, i}$ acts transitively on it for each $i$.

Proof. Given the dictionary between our setting and [B05] in the proof of Proposition 3.20 the first assertion of the proposition is a translation to our setting of B05, 4.B].

The second assertion of the proposition is a consequence of the first one and of Proposition 3.20

\section{Classification of Quasi-isolated elements}

The previous propositions make up together a method to classify quasi-isolated elements and their centralizers, which can be described as follows: 
- Describe the root datum $R(\sigma)$.

- Describe the quasi-isolated classes associated to this root datum using B05; by Proposition 3.21 these classes lift to quasi-isolated classes in $\mathbf{G} \cdot \sigma$.

- Determine $\mathbf{T}^{\sigma} /\left(\mathbf{T}^{\sigma}\right)^{0}$ using Lemma 1.2 ,

- Determine the type of the root datum of $\left(\mathbf{G}^{t \sigma}\right)^{0}$ either by Proposition 2.11 or an explicit computation when there are special orbits.

- Determine the isogeny type of $\left(\mathbf{G}^{t \sigma}\right)^{0}$ by computing its center explicitly.

- Determine $W(t \sigma)$ using that it can be computed in the datum $R(\sigma)$, see Proposition 3.14 .

We follow this method in the next subsections.

Quasi-isolated classes in type $A, n_{\sigma}=2$. We give the classification of quasiisolated and isolated classes of finite order in $\mathrm{GL}_{r} . \sigma, \mathrm{SL}_{r} . \sigma$ and $\mathrm{PGL}_{r} . \sigma$, where $\sigma$ is the quasi-central automorphism defined by $\sigma(g)=J^{t}\left(g^{-1}\right) J^{-1}$ where $J:=$ $\operatorname{antidiag}(\underbrace{1, \ldots, 1}_{\left\lfloor\frac{r}{2}\right\rfloor}, \underbrace{-1, \ldots,-1}_{\left\lfloor\frac{r+1}{2}\right\rfloor})$; here $\operatorname{antidiag}\left(x_{1}, \ldots, x_{r}\right)$ denotes the antidiagonal matrix with coefficient $x_{i}$ in the $i$-th column. Note that for $r=2$ the automorphism $\sigma$ acts trivially on $\mathrm{SL}_{2}$ and $\mathrm{PGL}_{2}$, while still defining on $\mathrm{GL}_{2}$ a conjugacy class of non-inner quasi-central automorphisms.

Proposition 4.1. If $p=2$ the class of $\sigma$ is the only quasi-isolated class in $\mathrm{GL}_{r} . \sigma$, $\mathrm{SL}_{r} . \sigma$ or $\mathrm{PGL}_{r} . \sigma$. In these groups the points over $k$ of the centralizer of $\sigma$ is $\operatorname{Sp}_{r}(k)$ if $r$ is even and $\mathrm{Sp}_{r-1}(k)$ if $r$ is odd.

Proof. The root datum $R(\sigma)$ is of type $B_{\left\lfloor\frac{r}{2}\right\rfloor}$ or $C_{\left\lfloor\frac{r}{2}\right\rfloor}$. For these types if $p=2$ one has $\left(\tilde{S}_{\sigma}\right)_{p^{\prime}}=\left\{\varpi_{s_{0}}^{\vee}\right\}$, whence the first assertion by Proposition 3.21. The second assertion comes from Proposition 1.30(5) and (6), using that in characteristic 2 all semisimple groups of type $C_{\left\lfloor\frac{r}{2}\right\rfloor}$ have same set of points over a field, and that in characteristic 2 , in type $A_{2 n}, \sigma$ is always of symplectic type.

Proposition 4.2. Assume $p \neq 2$; let $i$ be a primitive fourth root of unity, and for $j=0, \ldots,\left\lfloor\frac{r}{2}\right\rfloor \operatorname{let} t(j):=\operatorname{diag}(\underbrace{i, \ldots, i}_{j}, 1, \ldots, 1, \underbrace{-i, \ldots,-i}_{j}) \in \mathrm{SL}_{r}$. Then:

(1) The elements $t(j) . \sigma$ with $j=0, \ldots,\left\lfloor\frac{r}{2}\right\rfloor$ are representatives of the quasiisolated conjugacy classes in $\mathrm{GL}_{r} . \sigma, \mathrm{PGL}_{r} . \sigma$ for all $r$ and in $\mathrm{SL}_{r} . \sigma$ for odd $r$. They are all isolated, except in $\mathrm{GL}_{r}$ and $\mathrm{PGL}_{r}$ when $r$ is even and $j=1$. For odd $r$ and $\mathbf{G}=\mathrm{SL}_{r}$ or $\mathrm{PGL}_{r}$ (resp. $\mathbf{G}=\mathrm{GL}_{r}$ ) we have $C_{\mathbf{G}}(t(j) . \sigma)=$ $\mathrm{SO}_{2 j+1} \times \mathrm{Sp}_{r-1-2 j}\left(\right.$ resp. $\left.C_{\mathbf{G}}(t(j) \cdot \sigma)=\mathrm{O}_{2 j+1} \times \mathrm{Sp}_{r-1-2 j}\right)$.

For even $r$ and $\mathbf{G}=\mathrm{GL}_{r}$ (resp. $\mathbf{G}=\mathrm{PGL}_{r}$ ) we have $C_{\mathbf{G}}(t(j) . \sigma)=$ $\mathrm{O}_{2 j} \times \mathrm{Sp}_{r-2 j}$ (resp. $\left.\left(\mathrm{O}_{2 j} \times \mathrm{Sp}_{r-2 j}\right) / \pm 1\right)$.

(2) The elements $t(j) . \sigma$ with $j=0$ or $j=2, \ldots, r$ together with the element $-\sigma$ are representatives of the quasi-isolated conjugacy classes in $\mathrm{SL}_{2 r} . \sigma$. They are all isolated.

The centralizer of $t(j) . \sigma$ in $\mathrm{SL}_{2 r}$ is isomorphic to $\mathrm{SO}_{2 j} \times \mathrm{Sp}_{2 r-2 j}$.

In the above $\mathrm{SO}_{0}, \mathrm{O}_{0}$ and $\mathrm{SO}_{1}$ are trivial, $\mathrm{O}_{1}= \pm 1, \mathrm{SO}_{2}$ is a rank 1 torus and $\mathrm{O}_{2}$ is its extension by the automorphism $t \mapsto t^{-1}$ of the torus.

Proof. By Proposition 1.18 we already know that the quasi-isolated classes in $\mathrm{GL}_{r} . \sigma$ are in bijection with those of $\mathrm{PGL}_{r} . \sigma$. However the groups $\mathbf{T}^{\sigma} /\left(\mathbf{T}^{\sigma}\right)^{0}$ are different as we shall see below. 
We consider first the groups of type $A_{2 r}$, that is $\mathrm{GL}_{2 r+1} . \sigma, \mathrm{PGL}_{2 r+1} . \sigma$ and $\mathrm{SL}_{2 r+1} . \sigma$ with $\sigma$ quasi-central of symplectic type. By Proposition 2.4 the root datum $R(\sigma)$ is of type $C_{r}$ simply connected. We label its simple roots as $\bigcirc_{\alpha_{1}} \cdots \underset{\alpha_{2}}{\bigcirc} \alpha_{r-1}$ Using [B05, Proposition 4.9], we get that there are $r+1$ elements of $Y_{\sigma} \otimes \mathbb{Q}$ parameterizing quasi-isolated classes (all isolated) which are 0 and $\varpi_{j}^{\vee} / n_{j}$ where $\varpi_{j}^{\vee}$ runs over the fundamental coweights of $R(\sigma)$. For $1 \leq j \leq r-1$ we have $n_{j}=2$, and $n_{r}=1$. Let $\left\{\varpi_{j}^{\vee A} \mid j=1, \ldots, 2 r\right\}$ be the fundamental coweights in type $A_{2 r}$. The only special orbit of simple roots in $A_{2 r}$ corresponds to the coweights $\varpi_{r}^{\vee A}, \varpi_{r+1}^{\vee A}$, hence, by Proposition 2.3 we have $\varpi_{j}^{\vee}=\pi\left(\varpi_{j}^{\vee A}\right)$ for $j=1, \ldots, r-1$ and $\varpi_{r}^{\vee}=\frac{1}{2} \pi\left(\varpi_{r}^{\vee A}\right)$, so that the non-zero parameters of the quasi-isolated classes

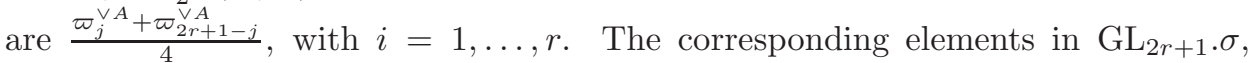
$\mathrm{PGL}_{2 r+1} . \sigma$ or $\mathrm{SL}_{2 r+1} . \sigma$ are the $t(j) . \sigma$ for $j=0, \ldots, r$. This proves the list of representatives in (1) for odd rank.

Consider now $\mathrm{SL}_{2 r} . \sigma$. By Proposition 2.4 the root datum $R(\sigma)$ is of type $B_{r}$ simply connected. We label the roots in $B_{r}$ similarly to $C_{r}$ with the double bond at the end. By [B05. Proposition 4.9] the parameters for quasi-isolated classes (all isolated) are 0 and $\varpi_{j}^{\vee} / n_{j}$, for $j=1, \ldots, r$. We have $n_{1}=1$ and $n_{j}=2$ for $n>1$. Since there is no special orbit of roots, we have $\varpi_{j}^{\vee}=\pi\left(\varpi_{j}^{\vee A}\right)$ for $j=1, \ldots, r$, so that the non-zero parameters for the quasi-isolated classes are $\frac{\varpi_{1}^{\vee A}+\varpi_{2 r-1}^{\vee A}}{2}$ and $\frac{\varpi_{j}^{\vee A}+\varpi_{2 r-j}^{\vee A}}{4}$ for $j=2, \ldots, r-1$ and $\frac{\varpi_{r}^{\vee A}}{2}$. The corresponding elements in $\mathrm{SL}_{2 r} . \sigma$ are respectively $\operatorname{diag}(-1,1,1, \ldots, 1,-1) . \sigma$, and $t(j) . \sigma$ for $j=2, \ldots, r$. This proves the list of representatives in (2).

Finally, consider now the groups $\mathrm{GL}_{2 r} . \sigma$ and $\mathrm{PGL}_{2 r} . \sigma$. By Proposition 2.4 the root datum $R(\sigma)$ is of type $B_{r}$ adjoint. If $r=1$ then $R(\sigma)$ is adjoint of type $B_{1}=A_{1}$. There is one non-zero parameter equal to $\varpi_{1}^{\vee}=\varpi_{1}^{\vee A}$. The corresponding class is not isolated. A representative is $\operatorname{diag}(1,-1) . \sigma$ (which is conjugate to $t(1) \cdot \sigma)$.

If $r>1$, by [B05, 4.E.1], the non-zero parameters for the quasi-isolated classes are $\frac{\varpi_{j}^{\vee}}{2}$ for $j=1, \ldots, r$, all corresponding to an isolated class except when $j=1$. Lifting to type $A$ we get $\frac{\varpi_{j}^{\vee, A}+\varpi_{2 r-j}^{\vee A}}{4}$ for $j=1, \ldots, r$. The corresponding elements are the $t(j) . \sigma$. This proves the representatives in (1) for even rank.

We compute now $C_{\mathbf{G}}(t(j) \sigma)$.

If $r$ is even, the matrix

$$
t(j) J=\operatorname{antidiag}(\underbrace{-i, \ldots,-i}_{j}, \underbrace{-1, \ldots,-1}_{r / 2-j}, \underbrace{1, \ldots, 1}_{r / 2-j}, \underbrace{-i, \ldots,-i}_{j})
$$

defines a bilinear form which is the orthogonal sum of a symmetric form on the first and last $j$ coordinates and an alternating form on the middle $r-2 j$ coordinates. Hence $C_{\mathbf{G}}(t(j) \sigma)$ is $\mathrm{O}_{2 j} \times \mathrm{Sp}_{r-2 j}$ when $\mathbf{G}=\mathrm{GL}_{r}$, is $\mathrm{SO}_{2 j} \times \mathrm{Sp}_{r-2 j}$ when $\mathbf{G}=\mathrm{SL}_{r}$ and is $\left(\mathrm{O}_{2 j} \times \mathrm{Sp}_{r-2 j}\right) / \pm 1$ when $\mathbf{G}=\mathrm{PGL}_{r}$ (the computation is the same in PGL since if $g$ preserves a bilinear form up to a scalar $\lambda$ then $\lambda^{-1 / 2} g$ preserves the form).

If $r$ is odd we have

$$
t(j) J=\operatorname{antidiag}(\underbrace{-i, \ldots,-i}_{j}, \underbrace{-1, \ldots,-1}_{(r-1) / 2-j},-1 \underbrace{1, \ldots, 1}_{(r-1) / 2-j}, \underbrace{-i, \ldots,-i}_{j}),
$$


hence it defines the orthogonal sum of a symmetric form on the first and last $j$ coordinates together with the $(r+1) / 2$-th coordinate and of an alternating form on the other coordinates. Hence $C_{\mathbf{G}}(t(j) \sigma)$ is $\mathrm{O}_{2 j+1} \times \mathrm{Sp}_{r-1-2 j}$ when $\mathbf{G}=\mathrm{GL}_{r}$, is $\mathrm{SO}_{2 j+1} \times \mathrm{Sp}_{r-1-2 j}$ when $\mathbf{G}=\mathrm{SL}_{r}$ and is $\left(\mathrm{O}_{2 j+1} \times \mathrm{Sp}_{r-1-2 j}\right) / \pm 1 \simeq \mathrm{SO}_{2 j+1} \times$ $\mathrm{Sp}_{r-1-2 j}$ when $\mathbf{G}=\mathrm{PGL}_{r}$.

Quasi-isolated classes in type $D, n_{\sigma}=2$. The semisimple groups of type $D_{n}$ which admit a quasi-central automorphism $\sigma$ with $n_{\sigma}=2$ are $\mathrm{PSO}_{2 n}, \mathrm{SO}_{2 n}$ and $\operatorname{Spin}_{2 n}$, see Proposition 1.30(iv).

Proposition 4.3. If $p=2$, the points over $k$ of a semisimple group $\mathbf{G}$ of type $D_{n}$ is always the group $\mathrm{SO}_{2 n}(k)$. The only quasi-isolated class of $\mathbf{G} \cdot \sigma$ is the class of $\sigma$, and $C_{\mathbf{G}}(\sigma) \simeq \mathrm{SO}_{2 n-1}(k)$.

Proof. The proposition results immediately from the fact that $R(\sigma)$ is of type $C_{n-1}$ by Proposition 2.4 thus has no non-central quasi-isolated elements by [B05, 5.B.2]; then $C_{\mathbf{G}}(\sigma)$ is described in Proposition 1.30 .

Assume now $p \neq 2$. Then $\mathrm{SO}_{2 n}$ is the special orthogonal group for the quadratic form given by the antidiagonal matrix of ones. An element of the diagonal maximal torus of $\mathrm{GL}_{2 n}$ is in $\mathrm{SO}_{2 n}$ if and only if its diagonal entries are of the form $t_{1}, \ldots, t_{n}, t_{n}^{-1}, \ldots, t_{1}^{-1}$. We shall denote by $\mathbf{T}$ the maximal torus of $\mathrm{SO}_{2 n}$, by $\operatorname{diag}\left(t_{1}, \ldots, t_{n}\right) \in \mathbf{T}$ such an element, and the effect of $\sigma$ on it is to replace $t_{n}$ with $t_{n}^{-1}$. If $\varepsilon_{1}, \ldots, \varepsilon_{n}$ is the corresponding basis of $Y(\mathbf{T})$, the simple roots are $\alpha_{1}=\varepsilon_{1}-\varepsilon_{2}, \ldots, \alpha_{n-1}=\varepsilon_{n-1}-\varepsilon_{n}, \alpha_{n}=\varepsilon_{n-1}+\varepsilon_{n}$ and the highest root is $\alpha_{0}=\varepsilon_{1}+\varepsilon_{2}$.

If $\left(x_{1}, \ldots, x_{n}\right)$ is an element of the maximal torus $\tilde{\mathbf{T}}$ of $\operatorname{Spin}_{2 n}$, where the coordinates correspond to the fundamental coweights, the quotient map $\tilde{\mathbf{T}} \rightarrow \mathbf{T}$ is given by $\left(x_{1}, \ldots, x_{n}\right) \mapsto \operatorname{diag}\left(x_{1}, \frac{x_{2}}{x_{1}}, \ldots, \frac{x_{n-2}}{x_{n-3}}, \frac{x_{n-1} x_{n}}{x_{n-2}}, \frac{x_{n}}{x_{n-1}}\right)$. The kernel of this map is generated by the central element $z:=(1, \ldots, 1,-1,-1)$. The effect of $\sigma$ is to exchange $x_{n-1}$ with $x_{n}$, so that $z \in[\tilde{\mathbf{T}}, \sigma]$.

Conversely $\operatorname{diag}\left(t_{1}, \ldots, t_{n}\right) \in \mathbf{T}$ can, if $\bar{t}$ is such that $\bar{t}^{2}=t_{1} \cdots t_{n}$, be lifted to $\left(t_{1}, t_{1} t_{2}, \ldots, t_{1} \cdots t_{n-2}, \bar{t} / t_{n}, \bar{t}\right) \in \tilde{\mathbf{T}}$. This gives two preimages differing by $z$, thus the conjugacy class in $\operatorname{Spin}_{2 n} \cdot \sigma$ of $\tilde{t} \sigma$, where $\tilde{t}$ is a lift of $\operatorname{diag}\left(t_{1}, \ldots, t_{n}\right) \in \mathbf{T}$, is uniquely defined.

Finally the maximal torus of $\mathrm{PSO}_{2 n}$ can be identified with $\mathbf{T} / \pm 1$.

Proposition 4.4. Assume $p \neq 2$. Let $t_{i}$ be the element $\operatorname{diag}(\underbrace{-1, \ldots,-1}_{i}, \underbrace{1, \ldots, 1}_{n-i})$ of $\mathrm{SO}_{2 n}$, and by abuse of notation still denote by $t_{i}$ one of its lifts to $\tilde{\mathbf{T}}$.

- The elements $\left\{t_{i} \sigma\right\}_{i=0, \ldots, n-1}$ are representatives of the $\mathbf{G}$-conjugacy classes of quasi-isolated elements of $\mathbf{G} \cdot \sigma$ for $\mathbf{G}=\mathrm{SO}_{2 n}$ and for $\mathbf{G}=\operatorname{Spin}_{2 n}$. These elements are isolated, and for $\mathbf{G}=\mathrm{SO}_{2 n}$ we have $C_{\mathbf{G}}\left(t_{i} \sigma\right) \simeq \mathrm{SO}_{2 i+1} \times$ $\mathrm{SO}_{2 n-2 i-1} \cdot \pm 1$. For $\mathbf{G}=\operatorname{Spin}_{2 n}$ we have $C_{\mathbf{G}}\left(t_{i} \sigma\right) \simeq\left(\operatorname{Spin}_{2 i+1} \times \operatorname{Spin}_{2 n-2 i-1}\right) /\left(z_{1}, z_{2}\right)$ where $z_{1}$ and $z_{2}$ are the generators of the center of the corresponding algebraic group (there is no quotient to take in the extreme case $i=0$ or $i=n-1$ where there is only one component).

- If $\mathbf{G}=\mathrm{PSO}_{2 n}$ then representatives of the isolated classes are $\sigma$ and the $\left\{t_{i} \sigma\right\}_{i=\left\lfloor\frac{n}{2}\right\rfloor, \ldots, n-2}$. We have $C_{\mathbf{G}}^{0}\left(t_{i} \sigma\right) \simeq \mathrm{SO}_{2 i+1} \times \mathrm{SO}_{2 n-2 i-1}$. The group 
$C_{\mathbf{G}}\left(t_{i} \sigma\right)$ is connected unless $i=\frac{n-1}{2}$ in which case $C_{\mathbf{G}}\left(t_{i} \sigma\right) / C_{\mathbf{G}}^{0}\left(t_{i} \sigma\right)$ is of order 2 and outer elements exchange the two components $C_{\mathbf{G}}^{0}\left(t_{i} \sigma\right)$, both isomorphic to $\mathrm{SO}_{n}$.

Let $u_{j}$ be the element $\operatorname{diag}(\underbrace{-1, \ldots,-1}_{j}, \underbrace{i, \ldots, i}_{n-1-2 j}, \underbrace{1, \ldots, 1}_{j+1})$. Representatives of the quasi-isolated classes which are not isolated are $\left\{u_{j} \sigma\right\}_{j=0, \ldots,\left\lfloor\frac{n}{2}\right\rfloor-1}$, and $C_{\mathbf{G}}^{0}\left(u_{j} \sigma\right)=\mathrm{GL}_{n-1-2 j} \times \mathrm{SO}_{2 j+1} \times \mathrm{SO}_{2 j+1}$. The group $C_{\mathbf{G}}\left(u_{j} \sigma\right) / C_{\mathbf{G}}^{0}\left(u_{j} \sigma\right)$ is of order 2 and outer elements act on $C_{\mathrm{G}}^{0}\left(u_{j} \sigma\right)$ by the outer automorphism of $\mathrm{GL}_{n-1-2 j}$ and the exchange of the two components $\mathrm{SO}_{2 j+1}$.

Proof. By Proposition 2.4 the root datum $R(\sigma)$ is of type $C_{n-1}$, simply connected when $\mathbf{G}$ is $\operatorname{Spin}_{2 n}$ or $\mathrm{SO}_{2 n}$, and adjoint when $\mathbf{G}$ is $\mathrm{PSO}_{2 n}$.

When $R(\sigma)$ is simply connected, by [B05, 4.10], representatives of quasi-isolated classes for $R(\sigma)$ (which are isolated) are $t_{i}^{\prime}=\operatorname{diag}(\underbrace{-1, \ldots,-1}_{i}, \underbrace{1, \ldots, 1}_{n-1-i})$; here we use a diag notation to describe the torus of $\mathrm{Sp}_{2 n-2}$ similar to the one we used for $\mathrm{SO}_{2 n}$. With these conventions, the map $\mathbf{T} \rightarrow \mathbf{T} /[\mathbf{T}, \sigma]$ consists in forgetting the last coordinate. The $t_{i}^{\prime}$ lift to $\left(\mathbf{T}^{\sigma}\right)^{0} \cdot \sigma$ as $t_{i} \sigma$, hence the representatives in this case.

When $R(\sigma)$ is adjoint, we use [B05, corollary 5.4], which contains the elements $t_{i}^{\prime}$ for $i=\left\lfloor\frac{n}{2}\right\rfloor, \ldots, n-1$ (taking into account that $t_{i}^{\prime}=-t_{i}^{\prime}$ since we are in the adjoint group). These lift to $\left(\mathbf{T}^{\sigma}\right)^{0} \cdot \sigma$ as the $\left\{t_{i} \sigma\right\}_{i=\left\lfloor\frac{n}{2}\right\rfloor, \ldots, n-2}$, except $t_{n-1}^{\prime}=1$ which lifts as $\sigma=t_{0} \sigma$. In the same corollary the quasi-isolated elements which are not isolated are given as the $u_{j}^{\prime}=\operatorname{diag}(\underbrace{-1, \ldots,-1}_{j}, \underbrace{i, \ldots, i}_{n-1-2 j}, \underbrace{1, \ldots, 1}_{j})$ which lift as the $u_{j} \sigma$.

We have thus verified the list of representatives in each case. Let us now determine $\mathbf{T}^{\sigma} /\left(\mathbf{T}^{\sigma}\right)^{0}$. By Lemma 1.2 2 (2) this group is trivial in the simply connected (resp. adjoint) case, since in this case $\sigma$ permutes a basis of $X$, the fundamental weights (resp. the simple roots). Remains the case of SO, where from our description we see that $\mathbf{T}^{\sigma}=\operatorname{diag}\left(t_{1}, \ldots, t_{n-1}, \pm 1\right)$ thus is not connected; $\mathbf{T}^{\sigma} /\left(\mathbf{T}^{\sigma}\right)^{0}$ is generated by -1 .

The next step is to compute $C_{\mathbf{G}}(t \sigma)$ for each representative $t$. We can use Proposition 2.11 to read off the type of $\Phi_{t \sigma}$ from [B05, Theorem 5.1(b) $(\alpha)$ ] and the values of $\Omega$ given in B05, example 4.10 and Corollary 5.4]. This confirms the type of the root system of $\left(\mathbf{G}^{\sigma}\right)^{0}$ given in the statement: for $t_{i}$ it is of type $B_{i} \times B_{n-i-1}$, and for $u_{j}$ of type $A_{n-2-2 j} \times B_{j} \times B_{j}$.

In order to describe the center of $\left(\mathbf{G}^{t \sigma}\right)^{0}$, let us describe the simple roots of this group. The simple roots of $R(\sigma)$ are $\beta_{1}:=\alpha_{1}, \ldots, \beta_{n-2}:=\alpha_{n-2}$ and $\beta_{n-1}:=\bar{\alpha}_{n}=$ $\alpha_{n-1}+\alpha_{n}$. The highest root of $R(\sigma)$ is $\beta_{0}:=\alpha_{1}+\alpha_{0}=\overline{\alpha_{1}+\ldots+\alpha_{n-1}}$. According to B05, corollary 5.4], the simple roots of $\Phi_{t_{i} \sigma}$ are $\beta_{0}, \ldots, \beta_{i-1}, \beta_{i+1}, \ldots, \beta_{n-1}$, thus the simple roots of $\Sigma_{t_{i} \sigma}$ consist of the same list where we replace $\beta_{0}$ by $\pi\left(\alpha_{1}+\right.$ $\left.\ldots+\alpha_{n-1}\right)$ and $\beta_{n-1}$ by $\pi\left(\alpha_{n}\right)$. In the basis $\varepsilon_{i}$, this can be stated as follows: if we set $\gamma_{0}:=\varepsilon_{1}, \gamma_{1}:=\varepsilon_{1}-\varepsilon_{2}, \ldots, \gamma_{n-2}:=\varepsilon_{n-2}-\varepsilon_{n-1}, \gamma_{n-1}:=\varepsilon_{n-1}$, the simple roots of $\Sigma_{t_{i} \sigma}$ are $\gamma_{0}, \ldots, \gamma_{i-1}, \gamma_{i+1}, \ldots, \gamma_{n-1}$. This enables us to compute the center of $C_{\mathbf{G}}^{0}\left(t_{i} \sigma\right)$ when $\mathbf{G}=\mathrm{SO}_{2 n}$, the kernel of the above set of roots in $\left(\mathbf{T}^{\sigma}\right)^{0}=\operatorname{diag}\left(x_{1}, \ldots, x_{n-1}, 1\right)$ which we find to be the trivial group. The same computation applies in $\mathbf{T} / \pm 1$. In $\tilde{\mathbf{T}}$ the center is reduced to $z$ which fits with the description we give. 
Similarly, the simple roots of $\Phi_{u_{j} \sigma}$ are $\gamma_{0}, \ldots, \gamma_{j-1}, \gamma_{j+1}, \ldots, \gamma_{n-2-j}, \gamma_{n-j}, \ldots, \gamma_{n-1}$. We find that the center of $C_{\mathbf{G}}^{0}\left(u_{j} \sigma\right)$ consists of the $\operatorname{diag}(\underbrace{1, \ldots, 1}_{j}, \underbrace{\lambda, \ldots, \lambda}_{n-1-2 j}, \underbrace{1, \ldots, 1}_{j})$, which justifies its description.

It remains to describe the elements of $\mathcal{A}_{t \sigma}$ (see Proposition 3.17). According to B05. Theorem 4.4(b)( $\beta)$ ] it consists of the elements of $\mathcal{A}_{R(\sigma)}$ which stabilize $\Phi_{t \sigma}$. The group $\mathcal{A}_{R(\sigma)}$ is generated by the element denoted by $z_{n}$ in [B05, table 1], equal to the longest element of the group generated by $s_{\beta_{1}}, \ldots, s_{\beta_{n-1}}$ times the longest element of the subgroup generated by $s_{\beta_{1}}, \ldots, s_{\beta_{n-2}}$. This involution maps $\beta_{i}$ to $\beta_{n-i-1}$; it stabilizes $\Phi_{t_{i} \sigma}$ if and only if $i=(n-1) / 2$, and stabilizes always $\Phi_{u_{j} \sigma}$. In each case it induces the action described in statement on $\Sigma_{t \sigma}$.

We now look at exceptional groups. We have extended the package CHEVIE (see M14) of GAP3 to implement the constructions of this paper. A coset $\mathbf{G} \cdot \sigma$ is represented as a CoxeterCoset, and the commands QuasiIsolatedRepresentatives and SemisimpleCentralizer have been extended to work with such cosets.

Quasi-isolated classes in type $E_{6}, n_{\sigma}=2$. The classification is the same for $\mathbf{G}$ of type $E_{6}$ adjoint or simply connected since the datum $R(\sigma)$, of type $F_{4}$, has a trivial connection index. Since $\mathbf{T}^{\sigma}=\left(\mathbf{T}^{\sigma}\right)^{0}$ by Lemma 1.2 (2), using Remark 3.19] it follows that quasi-semisimple elements have a connected centralizer, and that quasi-isolated elements are isolated.

The information in the next table can be obtained by the following CHEVIE commands:

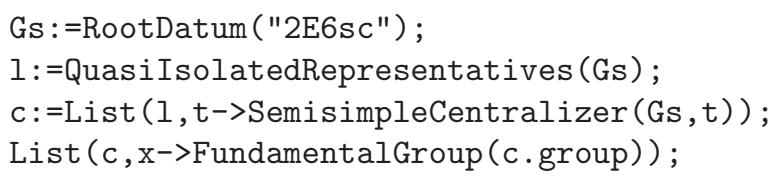

The first column of the next table lists representatives $t \in Q^{\vee} \otimes \mathbb{Q} / \mathbb{Z}$ such that t $\sigma$ runs over the quasi-isolated classes. The labelling of the diagram we use is<smiles>CC(CO)C(O)COCCO</smiles>

\begin{tabular}{|r|r|}
\hline representatives & $C_{G}(t \sigma)$ \\
\hline 0 & $F_{4}$ \\
$\alpha_{4}^{\vee} / 2$ & $\mathrm{PSp}_{8}$ \\
$\alpha_{2}^{\vee} / 2+\left(\alpha_{3}^{\vee}+\alpha_{5}^{\vee}\right) / 4$ & $\left(\mathrm{Spin}_{7} \times \mathrm{SL}_{2}\right) /\left(z_{1}, z_{2}\right)$ \\
$\left(2 \alpha_{2}^{\vee}+\alpha_{4}^{\vee}\right) / 3$ & $\left(\mathrm{SL}_{3} \times \mathrm{SL}_{3}\right) /\left(z_{1}, z_{2}\right)$ \\
$\left(3 \alpha_{2}^{\vee}+2 \alpha_{4}^{\vee}\right) / 4$ & $\left(\mathrm{SL}_{4} \times \mathrm{SL}_{2}\right) /\left(z_{1}, z_{2}\right)$ \\
\hline
\end{tabular}

In the above table $\left(z_{1}, z_{2}\right)$ means the element such that each component is a generator of the center of the corresponding algebraic group.

Quasi-isolated classes in type $D_{4}, n_{\sigma}=3$. The classification is the same for $\mathbf{G}$ adjoint or simply connected since the datum $R(\sigma)$, of type $G_{2}$, has a trivial connection index. Since $\mathbf{T}^{\sigma}=\left(\mathbf{T}^{\sigma}\right)^{0}$ by Lemma 1.2 (2), using Remark 3.19 it follows that quasi-semisimple elements have a connected centralizer, and that quasi-isolated elements are isolated. 
The information in the next table are obtained as for the above table, using this time "3D4sc" instead of "2E6sc". The labelling of the diagram we use is

\begin{tabular}{|r|r|}
\hline representatives & $C_{G}(t \sigma)$ \\
\hline 0 & $G_{2}$ \\
$\alpha_{3}^{\vee} / 2$ & $\mathrm{PGL}_{3}$ \\
$2 \alpha_{3}^{\vee} / 3$ & $\left(\mathrm{SL}_{2} \times \mathrm{SL}_{2}\right) /\left(z_{1}, z_{2}\right)$ \\
\hline
\end{tabular}

\section{REFERENCES}

[B05] C. Bonnafé, Quasi-isolated elements in reductive groups, Comm. Algebra 33 (2005), 2315-2337.

[Bbk] N. Bourbaki, Groupes et algèbres de Lie, chap IV-VI, Herman 1968.

[D84] D.I. Deriziotis, Conjugacy classes and centralizers of semisimple elements in finite groups of Lie type, Vorlesungen aus dem Fachbereich Mathematik der Universität Essen 11 (1984).

[DM91] F. Digne and J. Michel, Representations of finite groups of Lie type, London Mat. Soc. Student texts 21 (1991).

[DM94] F. Digne and J. Michel, Groupes réductifs non connexes, Annales de l'École Normale Supérieure 20 (1994), 345-406.

[DM02] F. Digne and J. Michel, Points fixes des automorphismes quasi-semi-simples, C.R.A.S. 334 (2001), 1055-1060.

[DM15] F. Digne and J. Michel, Complements on disconnected reductive groups, Pacific J. Math. 279 (2015), 203-228.

[M14] J. Michel, The development version of the CHEVIE package of GAP3., J. Algebra 435 (2015), 308-336.

[Sp06] T.A. Springer, Twisted conjugacy in simply connected groups, Transformation Groups 11 (2006), 539-545.

[St68] R. Steinberg, Endomorphisms of linear algebraic groups, Memoirs of the AMS 80 (1968). 
$\alpha_{0, i}, 14$

affine Weyl group, 14

$\bar{\alpha}, 8$

$\bar{\alpha}^{\vee}, 4$

$\mathcal{A}_{R(\sigma), 21}$

$\mathcal{A}_{t \sigma}, 21$

AZ, 19

$\mathcal{C}, 14$

$\mathcal{C}_{i}, 14$

$C_{\sigma, \mathcal{O}, 4}$

$\Delta_{\sigma}, 12$

$\tilde{\Delta}_{\sigma}, 14$

$\Delta_{t \sigma}, 18$

$e(G), 19$

$e(x), 19$

G, $\mathbf{G}^{1}, \mathbf{1}$

$\gamma_{s}, 14$

I, 14

isolated, 17

$J_{i}$, 14

minuscule, 16

$n_{s}, 14$

$n_{\sigma}, 1$

$\mathcal{O}(\alpha), 4$

orthogonal type, 9

$\varpi_{s}^{\vee}, 14$

$P, 12$

$p, 1$

$P^{\vee}, 13$

$P\left(\Phi_{\sigma}\right), 12$

$P\left(\Phi_{\sigma}^{\vee}\right), 14$

$P\left(\Sigma_{\sigma}\right), 10$

$\Phi_{\sigma}, \Phi_{\sigma}^{\vee}, 12$

$\Phi_{\sigma, i}, 14$
$\Phi_{t \sigma}, 15$

$\Pi, 4$

$\pi, 20$

$Q, 16$

$Q^{\vee}, 13$

$Q\left(\Phi_{\sigma}\right), 13$

$Q\left(\Phi_{\sigma}^{\vee}\right), 14$

$Q\left(\Sigma_{\sigma}\right), 10$

$Q\left(\Sigma_{\sigma}^{\vee}\right), 11$

quasi-central, 8

quasi-isolated, 20

$R(\sigma), 13$

$s_{0, i}, 14$

$\Sigma, \Sigma^{\vee}, 4$

$\Sigma_{\sigma}, \Sigma_{\sigma}^{\vee}, \Sigma_{t \sigma}, \Sigma_{t \sigma}^{\vee}$, 囵

$\Sigma_{\sigma}^{+}$, [5

special, cospecial, 4

$S_{\sigma, i}, 14$

$S_{t \sigma}, 18$

$\tilde{S}_{\sigma}, 14$

$\tilde{S}_{\sigma, i}, 14$

symplectic type, 9

$\mathbf{T}, \mathbf{T}^{1}, \mathbf{1}$

$[\mathbf{T}, \sigma], 3$

$W$, 1

$W(\sigma), W(t \sigma), 6$

$W^{0}(\sigma), W^{0}(t \sigma), 5$

$W_{a}, 14$

$W_{a}^{\prime}, 14$

$W_{i}^{\sigma}, 14$

$\widetilde{W}, 7$

$X, 2$

$X_{\sigma}, 3$

$Y$,2

$Y_{\sigma}, 3$

$z_{s}, 14$

(J. Michel) Institut de Mathématiques de Jussieu - Paris rive Gauche, Université Denis Diderot, Bâtiment Sophie Germain, 75013, Paris France.

E-mail address: jean.michel@imj-prg.fr

$U R L$ : webusers.imj-prg.fr/ jean.michel

(F. Digne) Laboratoire AmiÉnois de Mathématique Fondamentale et Appliquée, CNRS UMR 7352, Université de Picardie-Jules Verne, 80039 Amiens Cedex France.

E-mail address: digne@u-picardie.fr

$U R L$ : www.lamfa.u-picardie.fr/digne 